\title{
ARIMA Time Series Analysis in Forecasting Daily Stock Price of Chittagong Stock Exchange (CSE)
}

\author{
Tasnim Uddin Chowdhury ${ }^{1}$, Md. Shahidul Islam ${ }^{2}$ \\ ${ }^{1}$ Assistant Professor (Finance Discipline), Department of Business Administration, Premier University, Chattogram, Bangladesh, \\ ${ }^{2}$ Divisional Officer, Service Engineering Division, Bangladesh Forest Research Institute, Chattogram, Bangladesh
}

\begin{abstract}
The aim of the study is to examine the nature of daily share price and select a suitable ARIMA model to forecast the future daily share price from the previous daily share price of Chittagong Stock exchange (CSE). A random sampling method has been followed to collect the closing price of 60 companies for the period of January 2019 to December 2019 (241 trading days). Durbin-Watson test has been conducted to find the autocorrelation in each of the share prices. Then the Augmented Dickey-Fuller test has been applied to test the stationary of data and the Autocorrelation function (ACF) and Partial Autocorrelation function (PACF) has been calculated to determine the lag value of moving average $M A(q)$ and autocorrelation $A R(p)$ based on Ljung-Box Test $Q$, root mean square error, mean absolute error, mean absolute percent error and R-square values. After selecting ARIMA (p,d,q) model, forecasted values for each of the shares are calculated for the next 22 trading days of January 2020. Then a comparison has been made between the forecasted prices and the actual share prices by using the Goodness-of-fit Test, Root Mean Square Error (RMSE), Mean Absolute Percentage Error (MAPE), Mean Square Error (MSE) to validate the model. The result shows that the ARIMA model is applicable to forecast the daily share price of CSE.
\end{abstract}

Keywords-Time series analysis, Autoregressive Integrated Moving Average (ARIMA), Durbin-Watson test, Augmented Dickey-Fuller test, Goodness of fit test.

\section{BACKGROUND}

$\mathrm{S}$ tock market analysts prefer to conduct both fundamental analysis and technical analysis for taking investment decision. The former one involves analyzing the fundamental factors like- the company's financial position, operating performance, dividend payment history, market competition etc. that affects the future earning capacity of the company and identifying the mispriced stocks by determining its intrinsic value. On the contrary, the latter one undertakes the analysis of historical price movements to get a forecast of future price. To conduct technical analysis different charts like- bar chart, line chart, candle stick chart etc. is used. By using the charts different patterns of price movement is identified to get a sense of price trend that may prevail in the future. Jarrett and Kyper (2005) find predictable patterns in monthly stock prices examining the daily returns for more than 50 firms from American Stock Exchanges. But, unlike technical analysis, time series analysis is a statistical tool which is applied to forecast the price for a particular period of time in the future based on the historical series of data regarding price at constant time interval.

Forecasting involves taking sense of what may happen in the future based on the available past data. For the investors who actively trade in the stock market, forecasting the price of individual stock and market behavior based on past price movement plays a significant role in their decision making. The more accurate a forecast is; the more gain an investor would have. On the contrary, if the forecast goes wrong, it may even result in a significant loss. As such, stock market forecasting has been considered as one of most difficult tasks in the area of finance due to the stochastic behaviors and complex interdependencies of stock market (Wei, 2013).

\section{LITERATURE REVIEW}

A good number of researches have been done to predict the future movement patterns of stock prices in the literature of finance. Several studies find that, it is possible to forecast the stock price with high level of accuracy by applying statistical, econometric and machine learning models if the models can be formulated properly. Sen and Datta forecasted the stock price with a high level of accuracy based on time series decomposition (Sen \& Datta Chaudhuri, 2018a;Sen \& Datta Chaudhuri, 2018b; Sen \& Datta Chaudhuri, 2017a; Sen \& Datta Chaudhuri 2017b; Sen \& Data Chaudhuri,2017c; Sen \& Datta Chaudhuri, 2017d; Sen \& Datta Chaudhuri, 2016).Studies have also been done by Sohail, Kamal and Ali (2012), Kumar (2006) and Al-Zeaud (2011).

Another school of researchers support the "efficient market hypothesis" and belief that it is not possible to forecast the price of stock appropriately. Keane (1983) finds that the extent to which an emerging market would be inefficient depends on the market size, thinness of trading and quality of disclosed information. Poterba \& Summers (1988), Roux \& Gilberson (1978), Harvey (1994), Classens et al (1995), Khababa (1998) finds that the market is not efficient when it is in the weak form which implies that, there is possibility to predict the future stock prices and design a trading strategy which is profitable based on historical prices of stock. According to the studies of Fama and French (1988), Poterba and Summers (1988) and Ding, Granger and Engle(1993) all the time series of stock returns does not follow a random-walk process. Although, Kendall (1953), Granger \& Morgenstern 
(1963) and Solnik (1973) finds that random walk is usually valid for the stock markets of developed countries.

Applying the ARIMA $(1,0,1)$ for Egypt, $\operatorname{ARIMA}(1,0,2)$ for Ghana and ARIMA $(2,0,1)$ for Mauritius, Simons and Laryea (2004) predicts the stock market return and generates one period forecasts for the subsequent twelve periods. Al-Shihab (2006) examines the daily index of Amman Stock Exchange (ASE) for the period starting from April, 2004 toOctober, 2004 and tried to forecast the return of the market. Jia (2016) studies the effectiveness of Long Short-Term Memory (LSTM)for the prediction of stock market and finds the model to be effective for getting a pattern in the stock market.

In this age of digital era, forecasting the stock market through the use of Artificial Intelligence (AI) and different Machine Learning (ML) methods has also become a vital issue in the field of finance and investment. Over the decade, researchers have devoted their utmost effort to come up with predictive models which is more reliable (Ariyo et al., 2014). Lots of studies have been done to forecast the stock price by using AI and ML. To improve the time series forecasting several ML methods like- Support Vector Regression (SVR), Artificial Neural Networks (ANNs), Bayesian Neural Network (BNN) etc has been applied throughout the recent years (Wei, 2013). Neural Networks (NNs) have been proved to be one of the more important methods for predicting the stock price with better accuracy (Schöneburg, 1990).It requires very large number of historical data (Kryzanowski et al., 1993; Yoon et al., 1994) and in case of complicated networks, reliability of results may be low (Yoon et al., 1994). Mostafa (2010) used neural network-based models and predicted the stock market movements in Kuwait appropriately. Oh et al. (2006), Chang and Liu (2008) and Liu et al. (2012) works on fuzzy neural networks and Cao and Tay (2003), Gavrishchaka and Banerjee (2006)and Yeh et al. (2011) works on SVR model to overcome the shortcomings that traditional time series analysis tools have.

Study to forecast the stock pricehas also been conducted based on Dhaka Stock Exchange (DSE).Kader and Rahman(2005), Islam and Khaled(2005), Ahmed (2002); Rahman and Hossain(2006) and Alam and Uddin (2007) finds that the market is inefficient. So, their study supports that historical prices of the stocks can be used to forecast the future market returns in DSE. Rahman \& Hossain (2006) applies $\operatorname{ARIMA}(3,0,1)$ and $\operatorname{ARIMA}(1,0,1)$ models on the stock prices of companies from DSE and finds that the models are best fit for forecasting. Study has also been done by Mollick and Bepari (2008) using ARIMA(1,0,2). They also find applicability of the model they used in their study. Haider and Kabir (2009) applies $\operatorname{ARIMA}(3,1,1)$ and $\operatorname{ARMA}(3,1)$ to forecast the growth of the market based on the index and return series of DSE from the period of January 1993 to March 2011. Working with the monthly data of DSE general index for the period of January 2002 to July 2013, Kamruzzaman, Khudri and Rahman (2017) finds that, application of ARIMA $(2,0,2)$ is effective as a model to forecast the market return behavior of DSE.

Beside the aforementioned studies, lots of studies have also been performed to develop an appropriate model for forecasting the stock price. Most of the studies are out of the context of Bangladesh. Even, the models applied in the studies are very much difficult for the investors to understand and apply to forecast the price of stock. This study tries to examine whether time series model, a simple and convenient model for understanding, is applicable or not, to forecast the price of stock in the Chittagong Stock Exchange(Coordinate: $22.321079^{\circ} \mathrm{N} 91.811129^{0} \mathrm{E}$ ), one of the two major stock exchanges of Bangladesh.

\section{OBJECTIVES OF THE STUDY}

The major objective of the study is to examine the nature of daily share price and select a suitable ARIMA model to forecast the daily share price from the previous daily share price of Chittagong Stock exchange.

\section{HYPOTHESIS}

This study tests the following three hypotheses:

\section{Hypothesis 1}

Null Hypothesis, $\mathrm{H}_{0}$ : There are no autocorrelation in the share prices.

Alternative Hypothesis, $\mathrm{H}_{1}$ : There are autocorrelation in the share prices.

\section{Hypothesis 2}

Null Hypothesis, $\mathrm{H}_{0}$ : Stock values have a unit root.

Alternative Hypothesis, $\mathrm{H}_{1}$ : Stock values have no unit root.

Hypothesis 3

Null Hypothesis, $\mathrm{H}_{0}$ : There is no significant difference between the actual share price and the forecasted share price.

Alternative Hypothesis, $\mathrm{H}_{1}$ : There is a significant difference between the actual share price and the forecasted share price.

\section{METHODOLOGY}

To examine the applicability of time series method secondary source of data has been used. A random sampling method has been followed to collect the closing price of sixty randomly selected companies (out of three hundred thirty eight listed companies)from the website of CSE (www.cse.com.bd). A total number of 241 trading days for the period of January 2019to December 2019 has been collected for each of the selected companies. Then Durbin Watson test has been conducted for each company to observe the autocorrelation in the daily share prices. The Augmented Dickey Fuller Test has been conducted to test the unit root for each company data. Then the $1^{\text {st }}$ difference has been calculated from the previous data values to make the data stationary. After that, AutoRegressive Integrated Moving Average (ARIMA) has been 
developed based on the autocorrelation function (ACF) and partial autocorrelation function (PACF) of lag 16from the collected data. The Auto-regression with $\mathrm{p}$ lag $\mathrm{AR}(\mathrm{p})$ may be defined as equation (i):

$$
y_{t}=\mu+\sum_{i=1}^{p} \gamma_{i} y_{t-i}+\epsilon_{t} \ldots \ldots \ldots(i)
$$

Where, $y_{t}$ is the share price at time $\mathrm{t}, \mu$ is constant, $\gamma_{i}$ is the coefficient of lag variable, $y_{t-i}$ is the share price at time $(\mathrm{t}-\mathrm{i})$ and $\epsilon_{t}$ is the error term.

The Moving average with q lag MA(q) may be defined as equation (ii):

$$
y_{t}=\mu+\sum_{i=1}^{q} \theta_{i} y_{t-i}+\epsilon_{t} \ldots \ldots \ldots
$$

Where, $y_{t}$ is the share price at time $\mathrm{t}, \mu$ is constant, $\theta_{i}$ is the coefficient of lag variable, $y_{t-i}$ is the share price at time $(\mathrm{t}-\mathrm{i})$ and $\epsilon_{t}$ is the error term. Now autoregressive moving average ARMA (p,q) model combines both $\mathrm{p}$ autoregressive terms with q moving average which can be termed as equation (iii):
To develop the ARMA (p,q) model it requires the data to be stationary. To convert the data into stationary differencing $\mathrm{I}(\mathrm{d})$ is made. Where, $\mathrm{d}$ is the differencing made to make the data stationary. The model that we get then is ARIMA (p,d,q) with autoregressive $\mathrm{p}$ lag, differencing $\mathrm{d}$ to make stationary and moving average $\mathrm{q}$ lag. Then the selection of ARIMA (p,d,q) has been done from 241 trading days (January 2019 to December 2019) for future forecasting each share values. To test the fitness of the model Ljung-Box Test Q, Root Mean Square Error (RMSE), Mean Absolute Percentage Error (MAPE), Mean Absolute Error (MAE) and R-square value has been used. After that, by applying the selected ARIMA model, the forecasted share price ofnext 22 trading days in the month of January 2020 has been calculated. Then these calculated forecasted prices have been compared with the actual prices by using the Goodness-of-fit Test, Root Mean Square Error (RMSE), Mean Absolute Percentage Error (MAPE)and Mean Square Error (MSE) to validate the model. IBM SPSS Statistics 20and STATA 15 statistical package has been used to conduct the analysis.

\section{RESULT AND DISCUSSION}

The line diagram of daily closing share price for each company is shown below in Figure 1.

$$
y_{t}=\mu+\sum_{i=1}^{p} \gamma_{i} y_{t-i}+\sum_{i=1}^{q} \theta_{i} y_{t-i}+\epsilon_{t} \ldots(i i i)
$$

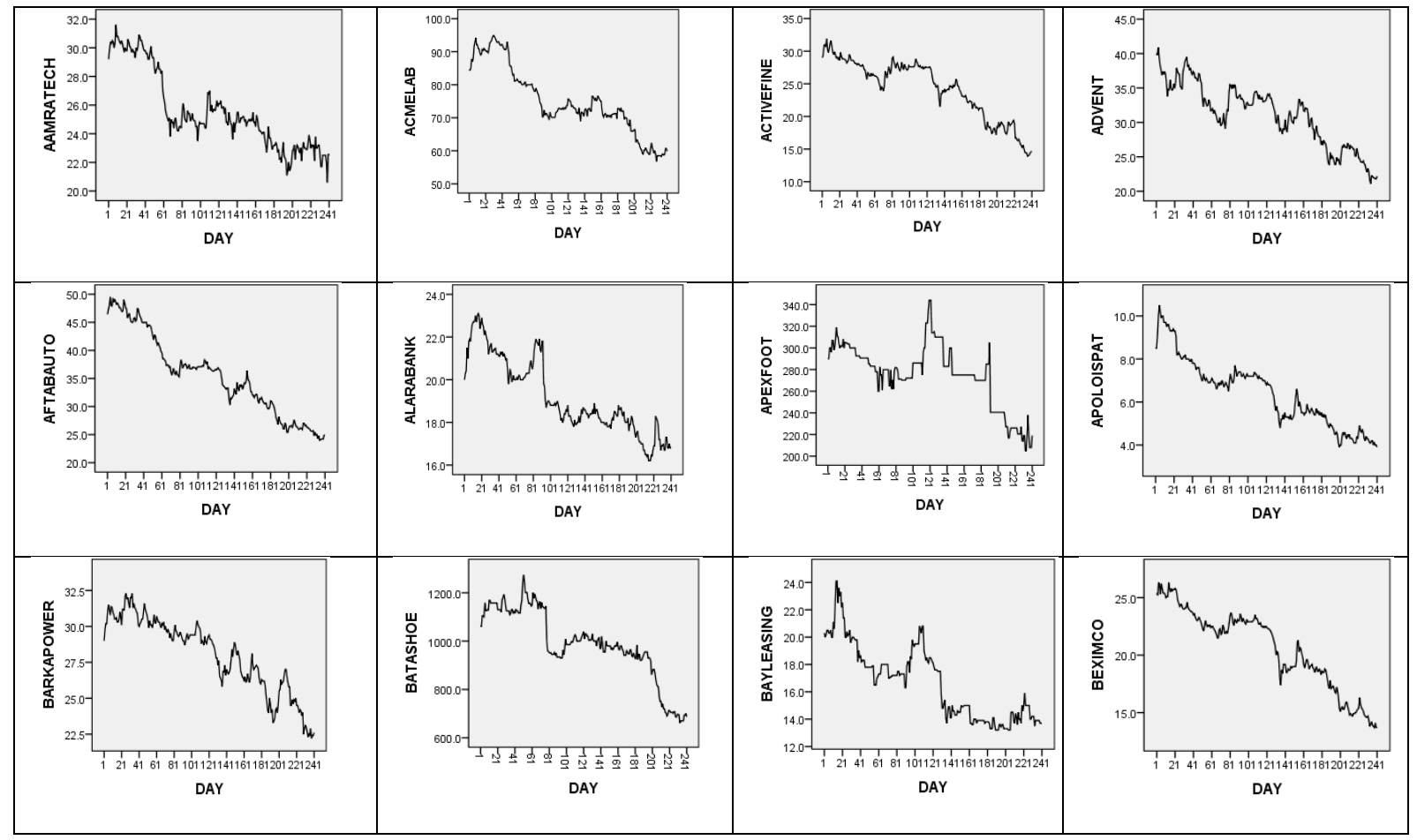




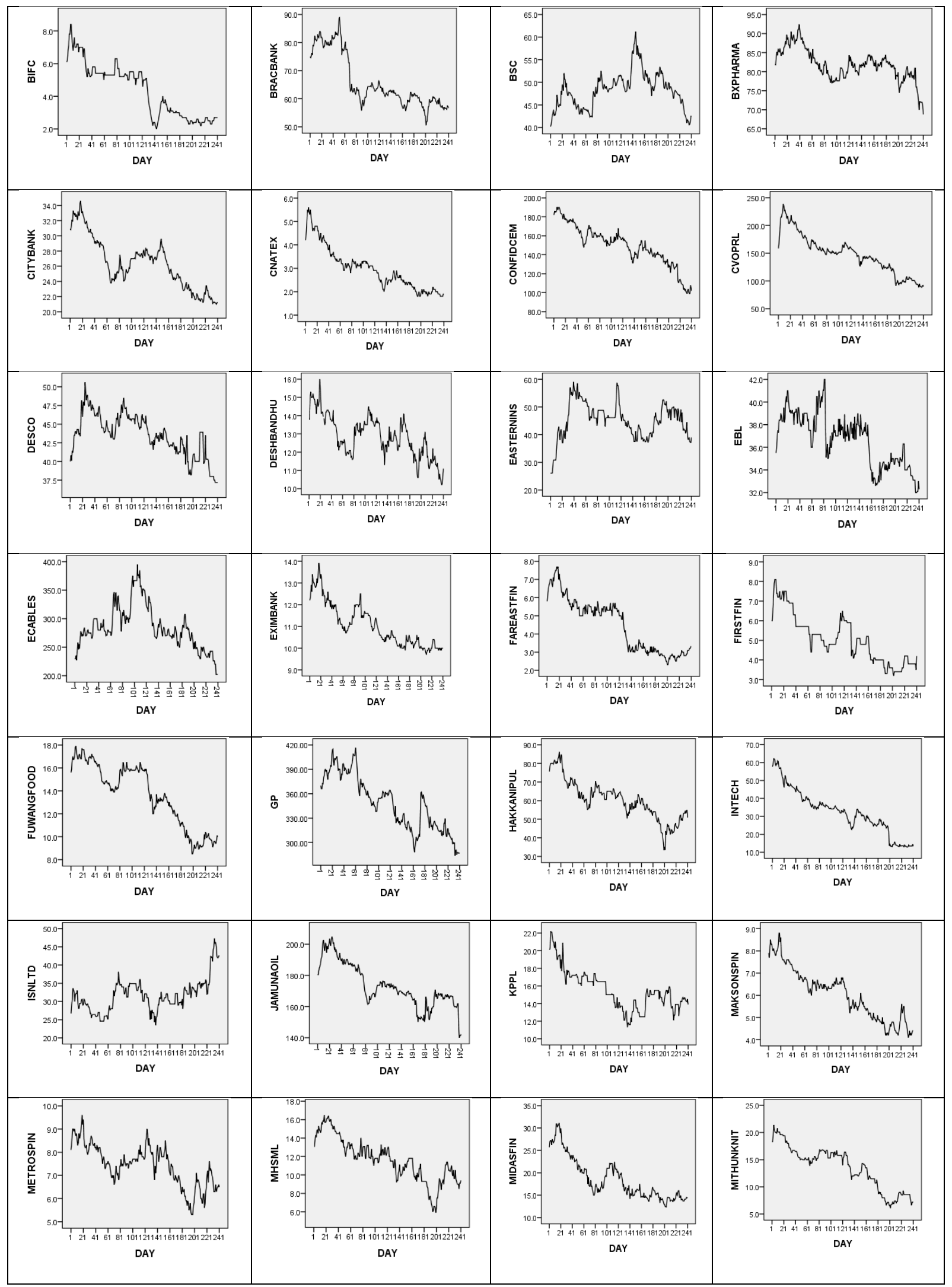




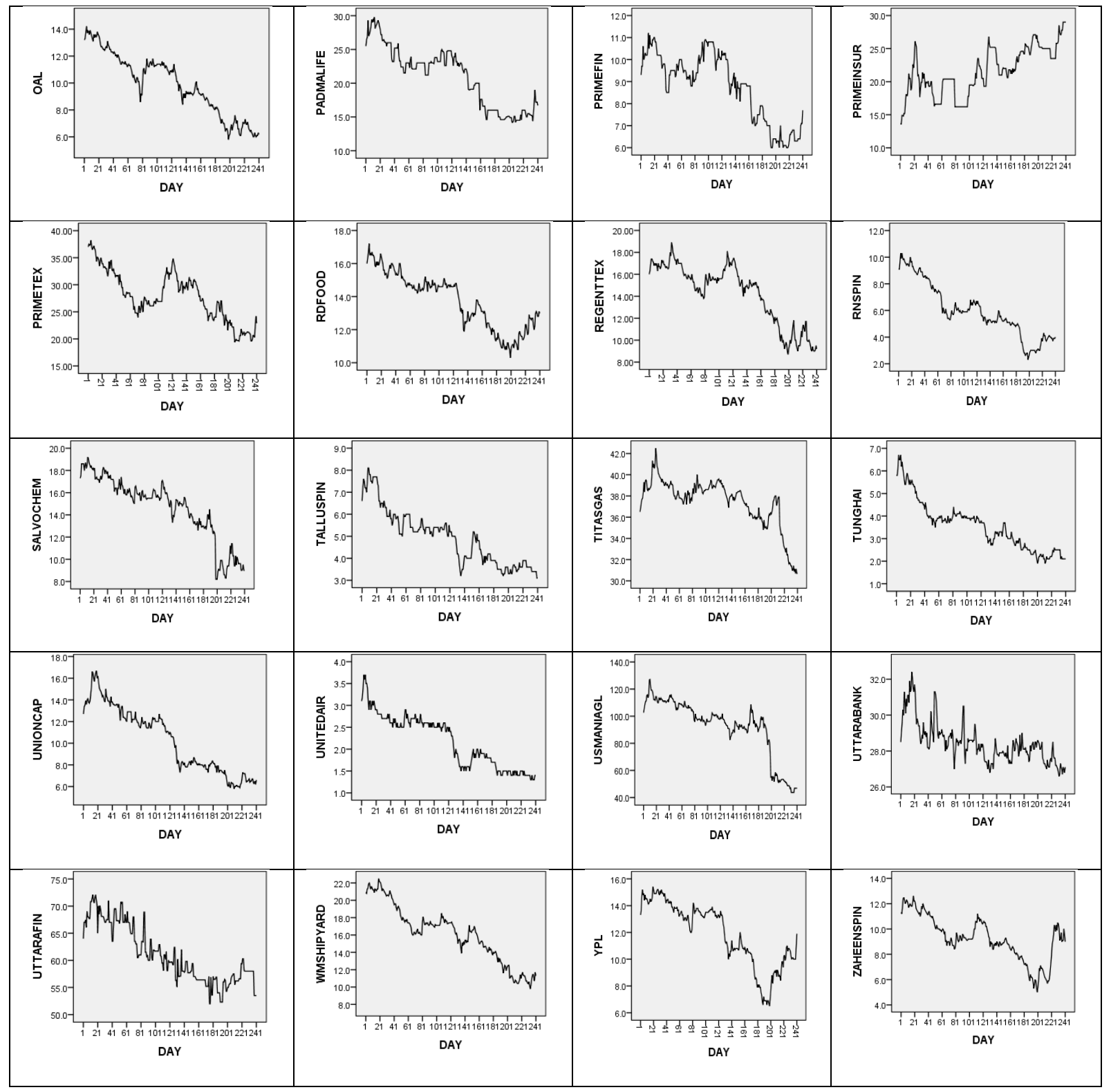

Figure 1: Line diagram of closing price

From the above figures, it is observed that all the shares have a trend value with constant intercept in y axis. The DurbinWatson test result for each share price is shown in Table 1. The Durbin-Watson test statistics of the share price varies from 0.065 to 0.462 . It is observed that all the test statistics values of Durbin-Watson test are nearly zero which rejects the null hypothesis 1 and provides an evidence of having autocorrelation in the daily share prices. The daily share prices of each company values are tested for unit root by using Augmented Dickey Fuller Test. The test statistic of Augmented Dickey-Fuller Test is shown in Table 1 for all the selected share prices. The test statistics $\mathrm{Z}(\mathrm{t})$ for Augmented Dickey-Fuller Test varies from 0.013 to 3.293 (with p-value 0.0152 to 0.9660$)$. In the case of EASTERNINS and UTTARABANK the p-value of Augmented Dickey-Fuller
Test are less than 0.05. Therefore, for the case of above two companies the null hypothesis 2 can be rejected which implies that the share prices of the companies are stationary. As a result, the share price of EASTERNINS and UTTARABANK are considered $\mathrm{d}$ equal to 0 in $\operatorname{ARIMA}(\mathrm{p}, \mathrm{d}, \mathrm{q})$ model. For rest of all 58 companies the p-value of Augmented Dickey-Fuller Test is greater than 0.05 which implies that, we can accept the null hypothesis 2 . So, it may be concluded that, for the 58 companies (96.67\%) the share price data are non-stationary. Now, the share values of the non-stationary 58 companies are deducted from the previous values ( $1^{\text {st }}$ differencing) of each company to make them stationary for applying the ARIMA model. As such, Augmented Dickey-Fuller Test has been conducted again for each company to test the stationarity of the data value and the result is shown in Table I. The test 
statistics for Augmented Dickey-Fuller Test for $1^{\text {st }}$ difference varies from 12.2490 to 25.8020 (with each p-value 0.0000). In these cases, the p-values are less than 0.05 . Now all the $1^{\text {st }}$ difference values are stationary and considered $\mathrm{d}$ equal to 1 for the 58 companies in ARIMA(p,d,q) model.

TABLE I Durbin Watson test, Augmented Dickey-Fuller Test and Model selection result

\begin{tabular}{|c|c|c|c|c|c|}
\hline $\begin{array}{l}\text { Name of the } \\
\text { Company }\end{array}$ & $\begin{array}{l}\text { Variable } \\
\text { Name }\end{array}$ & $\begin{array}{c}\text { Durbin } \\
\text { Watson } \\
\text { test } \\
\text { Statistic } \\
\quad \mathrm{s}\end{array}$ & $\begin{array}{c}\text { Stock } \\
\text { Price } \\
\text { Dickey } \\
\text {-Fuller } \\
\text { Z(t) } \\
\text { Statisti } \\
\text { c (p- } \\
\text { value) }\end{array}$ & $\begin{array}{c}1^{\text {st }} \\
\text { Differenc } \\
\mathrm{e} \\
\text { Dickey- } \\
\text { Fuller } \\
\text { Z(t) } \\
\text { Statistic } \\
\text { (p-value) }\end{array}$ & $\begin{array}{l}\text { Model } \\
\text { Select } \\
\text { ion }\end{array}$ \\
\hline $\begin{array}{c}\text { AAMRA } \\
\text { TECHNOLOGIES } \\
\text { LTD. }\end{array}$ & $\begin{array}{l}\text { AAMR } \\
\text { ATECH }\end{array}$ & 0.181 & $\begin{array}{l}1.574 \\
(0.496 \\
7)\end{array}$ & $\begin{array}{c}19.251 \\
(0.0000)\end{array}$ & $\begin{array}{c}\text { ARIM } \\
\text { A(1,1, } \\
0)\end{array}$ \\
\hline $\begin{array}{c}\text { THE ACME } \\
\text { LABORATORIES } \\
\text { LTD. }\end{array}$ & $\begin{array}{l}\text { ACME } \\
\text { LAB }\end{array}$ & 0.076 & $\begin{array}{c}0.390 \\
(0.911 \\
8)\end{array}$ & $\begin{array}{l}15.606 \\
(0.0000)\end{array}$ & $\begin{array}{c}\text { ARIM } \\
\text { A(1,1, } \\
0)\end{array}$ \\
\hline $\begin{array}{l}\text { ACTIVE FINE } \\
\text { CHEMICALS } \\
\text { LTD. } \\
\end{array}$ & $\begin{array}{l}\text { ACTIV } \\
\text { EFINE }\end{array}$ & 0.094 & $\begin{array}{c}0.015 \\
(0.957 \\
3) \\
\end{array}$ & $\begin{array}{c}14.495 \\
(0.0000)\end{array}$ & $\begin{array}{c}\text { ARIM } \\
\text { A(1,1, } \\
0)\end{array}$ \\
\hline $\begin{array}{c}\text { ADVENT } \\
\text { PHARMA LTD. }\end{array}$ & $\begin{array}{l}\text { ADVE } \\
\text { NT }\end{array}$ & 0.152 & $\begin{array}{c}1.325 \\
(0.617 \\
8)\end{array}$ & $\begin{array}{c}14.983 \\
(0.0000)\end{array}$ & $\begin{array}{c}\text { ARIM } \\
\text { A(1,1, } \\
0)\end{array}$ \\
\hline $\begin{array}{c}\text { AFTAB } \\
\text { AUTOMOBILES } \\
\text { LIMITED }\end{array}$ & $\begin{array}{l}\text { AFTAB } \\
\text { AUTO }\end{array}$ & 0.145 & $\begin{array}{c}0.738 \\
(0.836 \\
6)\end{array}$ & $\begin{array}{c}16.294 \\
(0.0000)\end{array}$ & $\begin{array}{c}\text { ARIM } \\
\text { A(1,1, } \\
0)\end{array}$ \\
\hline $\begin{array}{l}\text { AL-ARAFAH } \\
\text { ISLAMI BANK } \\
\text { LTD. }\end{array}$ & $\begin{array}{l}\text { ALARA } \\
\text { BANK }\end{array}$ & 0.160 & $\begin{array}{c}0.952 \\
(0.770 \\
3)\end{array}$ & $\begin{array}{c}16.722 \\
(0.0000)\end{array}$ & $\begin{array}{c}\text { ARIM } \\
\text { A(1,1, } \\
0)\end{array}$ \\
\hline $\begin{array}{c}\text { APEX } \\
\text { FOOTWEAR } \\
\text { LIMITED }\end{array}$ & $\begin{array}{l}\text { APEXF } \\
\text { OOT }\end{array}$ & 0.143 & $\begin{array}{c}1.654 \\
(0.455 \\
1)\end{array}$ & $\begin{array}{l}17.990 \\
(0.0000)\end{array}$ & $\begin{array}{c}\text { ARIM } \\
\text { A(1,1, } \\
0)\end{array}$ \\
\hline $\begin{array}{l}\text { APPOLLO ISPAT } \\
\text { COMPLEX LTD }\end{array}$ & $\begin{array}{l}\text { APOLO } \\
\text { ISPAT }\end{array}$ & 0.137 & $\begin{array}{c}0.743 \\
(0.835 \\
4)\end{array}$ & $\begin{array}{l}13.529 \\
(0.0000)\end{array}$ & $\begin{array}{c}\text { ARIM } \\
\text { A(1,1, } \\
0)\end{array}$ \\
\hline $\begin{array}{c}\text { BARAKA } \\
\text { POWER } \\
\text { LIMITED } \\
\end{array}$ & $\begin{array}{l}\text { BARK } \\
\text { APOW } \\
\text { ER } \\
\end{array}$ & 0.193 & $\begin{array}{c}0.350 \\
(0.918 \\
1) \\
\end{array}$ & $\begin{array}{l}16.243 \\
(0.0000)\end{array}$ & $\begin{array}{c}\text { ARIM } \\
\text { A(1,1, } \\
0) \\
\end{array}$ \\
\hline $\begin{array}{c}\text { BATA SHOE } \\
\text { COMPANY (BD) } \\
\text { LIMITED }\end{array}$ & $\begin{array}{l}\text { BATAS } \\
\text { HOE }\end{array}$ & 0.087 & $\begin{array}{c}0.143 \\
(0.945 \\
0)\end{array}$ & $\begin{array}{c}15.671 \\
(0.0000)\end{array}$ & $\begin{array}{c}\text { ARIM } \\
\text { A(1,1, } \\
0)\end{array}$ \\
\hline $\begin{array}{l}\text { BAY LEASING \& } \\
\text { INVESTMENT } \\
\text { LTD. }\end{array}$ & $\begin{array}{l}\text { BAYLE } \\
\text { ASING }\end{array}$ & 0.140 & $\begin{array}{c}1.559 \\
(0.503 \\
9) \\
\end{array}$ & $\begin{array}{c}17.236 \\
(0.0000)\end{array}$ & $\begin{array}{c}\text { ARIM } \\
\text { A(1,1, } \\
0) \\
\end{array}$ \\
\hline $\begin{array}{l}\text { BEXIMCO } \\
\text { LIMITED }\end{array}$ & $\begin{array}{l}\text { BEXIM } \\
\text { CO }\end{array}$ & 0.121 & $\begin{array}{c}0.235 \\
(0.934 \\
2)\end{array}$ & $\begin{array}{c}14.957 \\
(0.0000)\end{array}$ & $\begin{array}{c}\text { ARIM } \\
\text { A(1,1, } \\
0)\end{array}$ \\
\hline $\begin{array}{l}\text { BD INDUSTRIAL } \\
\text { FINANCE CO. } \\
\text { LTD }\end{array}$ & BIFC & 0.123 & $\begin{array}{c}1.062 \\
(0.730 \\
0)\end{array}$ & $\begin{array}{c}14.021 \\
(0.0000)\end{array}$ & $\begin{array}{c}\text { ARIM } \\
\text { A(1,1, } \\
0)\end{array}$ \\
\hline $\begin{array}{l}\text { BRAC BANK } \\
\text { LIMITED }\end{array}$ & $\begin{array}{l}\text { BRACB } \\
\text { ANK }\end{array}$ & 0.069 & $\begin{array}{c}1.073 \\
(0.725 \\
6)\end{array}$ & $\begin{array}{c}14.767 \\
(0.0000)\end{array}$ & $\begin{array}{c}\text { ARIM } \\
\text { A(1,1, } \\
0)\end{array}$ \\
\hline $\begin{array}{l}\text { BD SHIPPING } \\
\text { CORPORATION }\end{array}$ & BSC & 0.071 & $\begin{array}{c}2.286 \\
(0.176 \\
4)\end{array}$ & $\begin{array}{c}15.023 \\
(0.0000)\end{array}$ & $\begin{array}{c}\text { ARIM } \\
\text { A(1,1, } \\
0)\end{array}$ \\
\hline $\begin{array}{c}\text { BEXIMCO } \\
\text { PHARMACEUTIC } \\
\text { ALS LTD }\end{array}$ & $\begin{array}{l}\text { BXPHA } \\
\text { RMA }\end{array}$ & 0.147 & $\begin{array}{c}1.001 \\
(0.752 \\
8) \\
\end{array}$ & $\begin{array}{c}17.971 \\
(0.0000)\end{array}$ & $\begin{array}{c}\text { ARIM } \\
\text { A(2,1, } \\
0)\end{array}$ \\
\hline $\begin{array}{l}\text { THE CITY BANK } \\
\text { LIMITED }\end{array}$ & $\begin{array}{l}\text { CITYB } \\
\text { ANK }\end{array}$ & 0.065 & $\begin{array}{c}0.900 \\
(0.788 \\
0) \\
\end{array}$ & $\begin{array}{c}15.303 \\
(0.0000)\end{array}$ & $\begin{array}{c}\text { ARIM } \\
\text { A(1,1, } \\
0)\end{array}$ \\
\hline C \& A TEXTILES & CNATE & 0.126 & 1.136 & 15.361 & ARIM \\
\hline
\end{tabular}

\begin{tabular}{|c|c|c|c|c|c|}
\hline $\begin{array}{l}\text { Name of the } \\
\text { Company }\end{array}$ & $\begin{array}{l}\text { Variable } \\
\text { Name }\end{array}$ & $\begin{array}{c}\text { Durbin } \\
\text { Watson } \\
\text { test } \\
\text { Statistic } \\
\text { s }\end{array}$ & $\begin{array}{c}\text { Stock } \\
\text { Price } \\
\text { Dickey } \\
\text {-Fuller } \\
\text { Z(t) } \\
\text { Statisti } \\
\text { c (p- } \\
\text { value) }\end{array}$ & $\begin{array}{c}1^{\text {st }} \\
\text { Differenc } \\
\mathrm{e} \\
\text { Dickey- } \\
\text { Fuller } \\
\text { Z(t) } \\
\text { Statistic } \\
\text { (p-value) }\end{array}$ & $\begin{array}{l}\text { Model } \\
\text { Select } \\
\text { ion }\end{array}$ \\
\hline LIMITED & $\mathrm{X}$ & & $\begin{array}{c}(0.700 \\
6)\end{array}$ & $(0.0000)$ & $\begin{array}{c}\mathrm{A}(1,1, \\
0)\end{array}$ \\
\hline $\begin{array}{l}\text { CONFIDENCE } \\
\text { CEMENT } \\
\text { LIMITED }\end{array}$ & $\begin{array}{l}\text { CONFI } \\
\text { DCEM }\end{array}$ & 0.189 & $\begin{array}{l}0.650 \\
(0.859 \\
3)\end{array}$ & $\begin{array}{c}18.752 \\
(0.0000)\end{array}$ & $\begin{array}{c}\text { ARIM } \\
\text { A(1,1, } \\
0)\end{array}$ \\
\hline $\begin{array}{c}\text { CVO } \\
\text { PETROCHEMICA } \\
\text { L REFINERY } \\
\text { LIMITED }\end{array}$ & $\begin{array}{c}\text { CVOPR } \\
\mathrm{L}\end{array}$ & 0.123 & $\begin{array}{c}0.160 \\
(0.943 \\
1)\end{array}$ & $\begin{array}{l}12.249 \\
(0.0000)\end{array}$ & $\begin{array}{l}\text { ARIM } \\
\text { A(1,1, } \\
0)\end{array}$ \\
\hline $\begin{array}{c}\text { DHAKA } \\
\text { ELECTRIC } \\
\text { SUPPLY CO. } \\
\text { LTD. }\end{array}$ & DESCO & 0.217 & $\begin{array}{l}2.155 \\
(0.222 \\
9)\end{array}$ & $\begin{array}{c}17.945 \\
(0.0000)\end{array}$ & $\begin{array}{c}\text { ARIM } \\
\text { A(1,1, } \\
0)\end{array}$ \\
\hline $\begin{array}{l}\text { DESHBANDHU } \\
\text { POLYMER } \\
\text { LIMITED } \\
\end{array}$ & $\begin{array}{c}\text { DESHB } \\
\text { ANDH } \\
\text { U }\end{array}$ & 0.213 & $\begin{array}{c}2.275 \\
(0.180 \\
1)\end{array}$ & $\begin{array}{c}25.802 \\
(0.0000)\end{array}$ & $\begin{array}{c}\text { ARIM } \\
\text { A(1,1, } \\
0)\end{array}$ \\
\hline $\begin{array}{c}\text { EASTERN } \\
\text { INSURANCE } \\
\text { COMPANY LTD }\end{array}$ & $\begin{array}{l}\text { EASTE } \\
\text { RNINS }\end{array}$ & 0.091 & $\begin{array}{l}3.262 \\
(0.016 \\
7)\end{array}$ & & $\begin{array}{c}\text { ARIM } \\
\text { A(1,0, } \\
0)\end{array}$ \\
\hline $\begin{array}{l}\text { EASTERN BANK } \\
\text { LIMITED }\end{array}$ & EBL & 0.327 & $\begin{array}{c}2.462 \\
(0.125 \\
1) \\
\end{array}$ & $\begin{array}{c}19.061 \\
(0.0000)\end{array}$ & $\begin{array}{c}\text { ARIM } \\
\text { A(2,1, } \\
0)\end{array}$ \\
\hline $\begin{array}{l}\text { EASTERN } \\
\text { CABLES } \\
\text { LIMITED }\end{array}$ & $\begin{array}{l}\text { ECABL } \\
\text { ES }\end{array}$ & 0.073 & $\begin{array}{c}1.625 \\
(0.469 \\
9)\end{array}$ & $\begin{array}{c}15.188 \\
(0.0000)\end{array}$ & $\begin{array}{c}\text { ARIM } \\
\text { A(1,1, } \\
0)\end{array}$ \\
\hline $\begin{array}{c}\text { EXPORT } \\
\text { IMPORT BANK } \\
\text { OF BD LTD }\end{array}$ & $\begin{array}{l}\text { EXIMB } \\
\text { ANK }\end{array}$ & 0.172 & $\begin{array}{c}1.502 \\
(0.532 \\
4)\end{array}$ & $\begin{array}{l}17.450 \\
(0.0000)\end{array}$ & $\begin{array}{c}\text { ARIM } \\
\text { A(2,1, } \\
0)\end{array}$ \\
\hline $\begin{array}{c}\text { FAREAST } \\
\text { FINANCE \& } \\
\text { INVESTMENT } \\
\text { LIMITED } \\
\end{array}$ & $\begin{array}{l}\text { FAREA } \\
\text { STFIN }\end{array}$ & 0.160 & $\begin{array}{c}1.165 \\
(0.688 \\
6)\end{array}$ & $\begin{array}{c}20.050 \\
(0.0000)\end{array}$ & $\begin{array}{l}\text { ARIM } \\
\text { A(4,1, } \\
0)\end{array}$ \\
\hline $\begin{array}{l}\text { FIRST FINANCE } \\
\text { LIMITED }\end{array}$ & $\begin{array}{l}\text { FIRSTF } \\
\text { IN }\end{array}$ & 0.150 & $\begin{array}{c}1.483 \\
(0.542 \\
1)\end{array}$ & $\begin{array}{c}14.546 \\
(0.0000)\end{array}$ & $\begin{array}{c}\text { ARIM } \\
\text { A(1,1, } \\
0)\end{array}$ \\
\hline $\begin{array}{c}\text { FU-WANG } \\
\text { FOODS LIMITED }\end{array}$ & $\begin{array}{c}\text { FUWA } \\
\text { NGFOO } \\
\text { D }\end{array}$ & 0.087 & $\begin{array}{l}0.548 \\
(0.882 \\
3)\end{array}$ & $\begin{array}{c}15.264 \\
(0.0000)\end{array}$ & $\begin{array}{c}\text { ARIM } \\
\text { A(1,1, } \\
0)\end{array}$ \\
\hline $\begin{array}{l}\text { GRAMEENPHON } \\
\text { E LIMITED }\end{array}$ & GP & 0.144 & $\begin{array}{c}0.804 \\
(0.818 \\
0)\end{array}$ & $\begin{array}{l}14.294 \\
(0.0000)\end{array}$ & $\begin{array}{c}\text { ARIM } \\
\text { A(1,1, } \\
0)\end{array}$ \\
\hline $\begin{array}{l}\text { HAKKANI PULP } \\
\& \text { PAPER MILLS } \\
\text { LTD }\end{array}$ & $\begin{array}{l}\text { HAKK } \\
\text { ANIPU } \\
\text { L }\end{array}$ & 0.162 & $\begin{array}{c}1.710 \\
(0.425 \\
8)\end{array}$ & $\begin{array}{l}16.523 \\
(0.0000)\end{array}$ & $\begin{array}{c}\text { ARIM } \\
\text { A(1,1, } \\
0)\end{array}$ \\
\hline $\begin{array}{r}\text { INTECH } \\
\text { LIMITED }\end{array}$ & $\begin{array}{c}\text { INTEC } \\
\mathrm{H}\end{array}$ & 0.105 & $\begin{array}{c}1.342 \\
(0.609 \\
7)\end{array}$ & $\begin{array}{l}13.973 \\
(0.0000)\end{array}$ & $\begin{array}{c}\text { ARIM } \\
\text { A(1,1, } \\
0)\end{array}$ \\
\hline $\begin{array}{l}\text { INFORMATION } \\
\text { SERVICES } \\
\text { NETWORK LTD. }\end{array}$ & $\begin{array}{l}\text { ISNLT } \\
\text { D }\end{array}$ & 0.127 & $\begin{array}{c}1.789 \\
(0.386 \\
0)\end{array}$ & $\begin{array}{c}16.546 \\
(0.0000)\end{array}$ & $\begin{array}{c}\text { ARIM } \\
\text { A(1,1, } \\
0)\end{array}$ \\
\hline $\begin{array}{l}\text { JAMUNA OIL } \\
\text { COMPANY } \\
\text { LIMITED }\end{array}$ & $\begin{array}{l}\text { JAMUN } \\
\text { AOIL }\end{array}$ & 0.119 & $\begin{array}{c}0.553 \\
(0.881 \\
4)\end{array}$ & $\begin{array}{l}15.186 \\
(0.0000)\end{array}$ & $\begin{array}{c}\text { ARIM } \\
\text { A(2,1, } \\
0)\end{array}$ \\
\hline $\begin{array}{c}\text { KHULNA } \\
\text { PRINTING \& } \\
\text { PACKAGING } \\
\text { LIMITED } \\
\end{array}$ & KPPL & 0.126 & $\begin{array}{c}2.369 \\
(0.150 \\
8)\end{array}$ & $\begin{array}{c}16.799 \\
(0.0000)\end{array}$ & $\begin{array}{l}\text { ARIM } \\
\text { A(1,1, } \\
0)\end{array}$ \\
\hline $\begin{array}{c}\text { MAKSONS } \\
\text { SPINNING } \\
\text { MILLS LIMITED }\end{array}$ & $\begin{array}{l}\text { MAKS } \\
\text { ONSPI } \\
\mathrm{N}\end{array}$ & 0.211 & $\begin{array}{c}1.230 \\
(0.660 \\
6)\end{array}$ & $\begin{array}{c}15.501 \\
(0.0000)\end{array}$ & $\begin{array}{c}\text { ARIM } \\
\text { A(1,1, } \\
0)\end{array}$ \\
\hline $\begin{array}{c}\text { METRO } \\
\text { SPINNING }\end{array}$ & $\begin{array}{l}\text { METR } \\
\text { OSPIN }\end{array}$ & 0.167 & $\begin{array}{l}2.070 \\
(0.256 \\
\end{array}$ & $\begin{array}{c}15.611 \\
(0.0000)\end{array}$ & $\begin{array}{l}\text { ARIM } \\
\text { A }(1,1,\end{array}$ \\
\hline
\end{tabular}

Page 219 


\begin{tabular}{|c|c|c|c|c|c|}
\hline $\begin{array}{l}\text { Name of the } \\
\text { Company }\end{array}$ & $\begin{array}{l}\text { Variable } \\
\text { Name }\end{array}$ & $\begin{array}{l}\text { Durbin } \\
\text { Watson } \\
\text { test } \\
\text { Statistic } \\
\quad \mathrm{s}\end{array}$ & $\begin{array}{c}\text { Stock } \\
\text { Price } \\
\text { Dickey } \\
\text {-Fuller } \\
\text { Z(t) } \\
\text { Statisti } \\
\text { c (p- } \\
\text { value) } \\
\end{array}$ & $\begin{array}{c}1^{\text {st }} \\
\text { Differenc } \\
\text { e } \\
\text { Dickey- } \\
\text { Fuller } \\
\text { Z(t) } \\
\text { Statistic } \\
\text { (p-value) } \\
\end{array}$ & $\begin{array}{c}\text { Model } \\
\text { Select } \\
\text { ion }\end{array}$ \\
\hline LIMITED & & & 9) & & 0) \\
\hline $\begin{array}{c}\text { MOZAFFAR } \\
\text { HOSSAIN } \\
\text { SPINNING } \\
\text { MILLS LIMITED } \\
\end{array}$ & $\begin{array}{l}\text { MHSM } \\
\text { L }\end{array}$ & 0.176 & $\begin{array}{l}1.548 \\
(0.509 \\
9)\end{array}$ & $\begin{array}{c}16.329 \\
(0.0000)\end{array}$ & $\begin{array}{c}\text { ARIM } \\
\text { A(1,1, } \\
0)\end{array}$ \\
\hline $\begin{array}{c}\text { MIDAS } \\
\text { FINANCING } \\
\text { LIMITED } \\
\end{array}$ & $\begin{array}{l}\text { MIDAS } \\
\text { FIN }\end{array}$ & 0.105 & $\begin{array}{c}1.603 \\
(0.481 \\
9) \\
\end{array}$ & $\begin{array}{c}17.916 \\
(0.0000)\end{array}$ & $\begin{array}{c}\text { ARIM } \\
\text { A(1,1, } \\
0)\end{array}$ \\
\hline $\begin{array}{c}\text { MITHUN } \\
\text { KNITTING AND } \\
\text { DYEING (CEPZ) } \\
\text { LTD. } \\
\end{array}$ & $\begin{array}{l}\text { MITHU } \\
\text { NKNIT }\end{array}$ & 0.093 & $\begin{array}{l}0.585 \\
(0.874 \\
4)\end{array}$ & $\begin{array}{c}15.459 \\
(0.0000)\end{array}$ & $\begin{array}{l}\text { ARIM } \\
\text { A(1,1, } \\
0)\end{array}$ \\
\hline $\begin{array}{l}\text { OLYMPIC } \\
\text { ACCESSORIES } \\
\text { LTD. } \\
\end{array}$ & OAL & 0.158 & $\begin{array}{c}0.751 \\
(0.833 \\
2) \\
\end{array}$ & $\begin{array}{c}16.248 \\
(0.0000)\end{array}$ & $\begin{array}{c}\text { ARIM } \\
\text { A(1,1, } \\
0)\end{array}$ \\
\hline $\begin{array}{c}\text { PADMA ISLAMI } \\
\text { LIFE } \\
\text { INSURANCE } \\
\text { LIMITED } \\
\end{array}$ & $\begin{array}{l}\text { PADM } \\
\text { ALIFE }\end{array}$ & 0.151 & $\begin{array}{c}1.256 \\
(0.649 \\
0)\end{array}$ & $\begin{array}{c}17.236 \\
(0.0000)\end{array}$ & $\begin{array}{l}\text { ARIM } \\
\text { A(1,1, } \\
0)\end{array}$ \\
\hline $\begin{array}{c}\text { PRIME FINANCE } \\
\& \text { INVESTMENT } \\
\text { LTD }\end{array}$ & $\begin{array}{l}\text { PRIME } \\
\text { FIN }\end{array}$ & 0.114 & $\begin{array}{c}1.386 \\
(0.589 \\
1) \\
\end{array}$ & $\begin{array}{c}17.498 \\
(0.0000)\end{array}$ & $\begin{array}{c}\text { ARIM } \\
\text { A(1,1, } \\
0)\end{array}$ \\
\hline $\begin{array}{c}\text { PRIME } \\
\text { INSURANCE } \\
\text { COMPANY LTD }\end{array}$ & $\begin{array}{l}\text { PRIMEI } \\
\text { NSUR }\end{array}$ & 0.121 & $\begin{array}{l}1.913 \\
(0.325 \\
8)\end{array}$ & $\begin{array}{c}13.244 \\
(0.0000)\end{array}$ & $\begin{array}{l}\text { ARIM } \\
\text { A(1,1, } \\
0)\end{array}$ \\
\hline $\begin{array}{l}\text { PRIME TEXTILE } \\
\text { SPINNING } \\
\text { MILLS } \\
\end{array}$ & $\begin{array}{l}\text { PRIME } \\
\text { TEX }\end{array}$ & 0.086 & $\begin{array}{c}0.428 \\
(0.905 \\
3) \\
\end{array}$ & $\begin{array}{c}15.151 \\
(0.0000)\end{array}$ & $\begin{array}{c}\text { ARIM } \\
\text { A(1,1, } \\
0) \\
\end{array}$ \\
\hline $\begin{array}{c}\text { RANGPUR } \\
\text { DAIRY \& FOOD } \\
\text { PRODUCTS LTD }\end{array}$ & $\begin{array}{c}\text { RDFOO } \\
\text { D }\end{array}$ & 0.144 & $\begin{array}{c}1.564 \\
(0.501 \\
8) \\
\end{array}$ & $\begin{array}{c}15.779 \\
(0.0000)\end{array}$ & $\begin{array}{c}\text { ARIM } \\
\text { A(1,1, } \\
0)\end{array}$ \\
\hline $\begin{array}{c}\text { REGENT } \\
\text { TEXTILE MILLS } \\
\text { LIMITED } \\
\end{array}$ & $\begin{array}{l}\text { REGEN } \\
\text { TTEX }\end{array}$ & 0.084 & $\begin{array}{c}0.428 \\
(0.905 \\
3) \\
\end{array}$ & $\begin{array}{c}15.151 \\
(0.0000)\end{array}$ & $\begin{array}{c}\text { ARIM } \\
\text { A(1,1, } \\
0)\end{array}$ \\
\hline $\begin{array}{l}\text { R. N. SPINNING } \\
\text { MILLS LIMITED }\end{array}$ & $\begin{array}{l}\text { RNSPI } \\
\mathrm{N}\end{array}$ & 0.075 & $\begin{array}{c}1.243 \\
(0.654 \\
9) \\
\end{array}$ & $\begin{array}{c}14.700 \\
(0.0000)\end{array}$ & $\begin{array}{c}\text { ARIM } \\
\text { A(1,1, } \\
0)\end{array}$ \\
\hline SALVO & SALVO & 0.196 & 0.778 & 16.435 & ARIM \\
\hline
\end{tabular}

\begin{tabular}{|c|c|c|c|c|c|}
\hline $\begin{array}{l}\text { Name of the } \\
\text { Company }\end{array}$ & $\begin{array}{l}\text { Variable } \\
\text { Name }\end{array}$ & $\begin{array}{c}\text { Durbin } \\
\text { Watson } \\
\text { test } \\
\text { Statistic } \\
\text { s }\end{array}$ & $\begin{array}{c}\text { Stock } \\
\text { Price } \\
\text { Dickey } \\
\text {-Fuller } \\
\text { Z(t) } \\
\text { Statisti } \\
\text { c (p- } \\
\text { value) }\end{array}$ & $\begin{array}{c}1^{\text {st }} \\
\text { Differenc } \\
\mathrm{e} \\
\text { Dickey- } \\
\text { Fuller } \\
\text { Z(t) } \\
\text { Statistic } \\
\text { (p-value) }\end{array}$ & $\begin{array}{c}\text { Model } \\
\text { Select } \\
\text { ion }\end{array}$ \\
\hline $\begin{array}{c}\text { CHEMICAL } \\
\text { INDUSTRY LTD }\end{array}$ & CHEM & & $\begin{array}{c}(0.825 \\
5)\end{array}$ & $(0.0000)$ & $\begin{array}{c}\mathrm{A}(1,1, \\
0)\end{array}$ \\
\hline $\begin{array}{c}\text { TALLU } \\
\text { SPINNING } \\
\text { MILLS LIMITED }\end{array}$ & $\begin{array}{l}\text { TALLU } \\
\text { SPIN }\end{array}$ & 0.167 & $\begin{array}{c}1.144 \\
(0.697 \\
2)\end{array}$ & $\begin{array}{c}14.507 \\
(0.0000)\end{array}$ & $\begin{array}{c}\text { ARIM } \\
\text { A }(1,1, \\
0)\end{array}$ \\
\hline $\begin{array}{c}\text { TITAS GAS } \\
\text { TRANSMISSION } \\
\& \\
\text { DISTRIBUTION } \\
\text { CO.LTD }\end{array}$ & $\begin{array}{l}\text { TITAS } \\
\text { GAS }\end{array}$ & 0.085 & $\begin{array}{c}0.013 \\
(0.957 \\
4)\end{array}$ & $\begin{array}{l}16.175 \\
(0.0000)\end{array}$ & $\begin{array}{l}\text { ARIM } \\
\text { A }(1,1, \\
0)\end{array}$ \\
\hline $\begin{array}{c}\text { TUNG HAI } \\
\text { KNITTING AND } \\
\text { DYEING LTD. }\end{array}$ & $\begin{array}{l}\text { TUNG } \\
\text { HAI }\end{array}$ & 0.133 & $\begin{array}{c}1.622 \\
(0.471 \\
6) \\
\end{array}$ & $\begin{array}{c}17.318 \\
(0.0000)\end{array}$ & $\begin{array}{c}\text { ARIM } \\
\text { A }(1,1, \\
0)\end{array}$ \\
\hline $\begin{array}{l}\text { UNION CAPITAL } \\
\text { LIMITED }\end{array}$ & $\begin{array}{l}\text { UNION } \\
\text { CAP }\end{array}$ & 0.168 & $\begin{array}{c}0.639 \\
(0.862 \\
0)\end{array}$ & $\begin{array}{l}16.470 \\
(0.0000)\end{array}$ & $\begin{array}{c}\text { ARIM } \\
\text { A(1,1, } \\
0)\end{array}$ \\
\hline $\begin{array}{c}\text { UNITED } \\
\text { AIRWAYS (BD) } \\
\text { LTD. }\end{array}$ & $\begin{array}{l}\text { UNITE } \\
\text { DAIR }\end{array}$ & 0.197 & $\begin{array}{c}1.295 \\
(0.631 \\
7)\end{array}$ & $\begin{array}{c}18.205 \\
(0.0000)\end{array}$ & $\begin{array}{c}\text { ARIM } \\
\text { A }(1,1, \\
0)\end{array}$ \\
\hline $\begin{array}{c}\text { USMANIA } \\
\text { GLASS SHEET } \\
\text { FACTORY } \\
\text { LIMITED }\end{array}$ & $\begin{array}{l}\text { USMA } \\
\text { NIAGL }\end{array}$ & 0.067 & $\begin{array}{c}0.099 \\
(0.966 \\
0)\end{array}$ & $\begin{array}{c}14.823 \\
(0.0000)\end{array}$ & $\begin{array}{l}\text { ARIM } \\
\text { A }(1,1, \\
0)\end{array}$ \\
\hline $\begin{array}{l}\text { UTTARA BANK } \\
\text { LIMITED }\end{array}$ & $\begin{array}{c}\text { UTTAR } \\
\text { ABAN } \\
\text { K }\end{array}$ & 0.391 & $\begin{array}{c}3.293 \\
(0.015 \\
2)\end{array}$ & & $\begin{array}{c}\text { ARIM } \\
\text { A }(2,0, \\
0)\end{array}$ \\
\hline $\begin{array}{c}\text { UTTARA } \\
\text { FINANCE \& } \\
\text { INVESTMENT } \\
\text { LIMITED }\end{array}$ & $\begin{array}{l}\text { UTTAR } \\
\text { AFIN }\end{array}$ & 0.462 & $\begin{array}{c}2.332 \\
(0.162 \\
0)\end{array}$ & $\begin{array}{c}18.772 \\
(0.0000)\end{array}$ & $\begin{array}{l}\text { ARIM } \\
\text { A }(3,1, \\
0)\end{array}$ \\
\hline $\begin{array}{c}\text { WESTERN } \\
\text { MARINE } \\
\text { SHIPYARD LTD. }\end{array}$ & $\begin{array}{l}\text { WMSHI } \\
\text { PYARD }\end{array}$ & 0.122 & $\begin{array}{c}0.838 \\
(0.807 \\
8)\end{array}$ & $\begin{array}{l}15.386 \\
(0.0000)\end{array}$ & $\begin{array}{c}\text { ARIM } \\
\text { A(1,1, } \\
0)\end{array}$ \\
\hline $\begin{array}{c}\text { YEAKIN } \\
\text { POLYMER } \\
\text { LIMITED } \\
\end{array}$ & YPL & 0.073 & $\begin{array}{c}1.129 \\
(0.703 \\
5) \\
\end{array}$ & $\begin{array}{l}13.556 \\
(0.0000)\end{array}$ & $\begin{array}{c}\text { ARIM } \\
\text { A }(1,1, \\
0)\end{array}$ \\
\hline $\begin{array}{c}\text { ZAHEEN } \\
\text { SPINNING LTD. }\end{array}$ & $\begin{array}{l}\text { ZAHEE } \\
\text { NSPIN }\end{array}$ & 0.066 & $\begin{array}{c}1.566 \\
(0.500 \\
5)\end{array}$ & $\begin{array}{c}14.117 \\
(0.0000)\end{array}$ & $\begin{array}{c}\text { ARIM } \\
\text { A }(1,1, \\
0)\end{array}$ \\
\hline
\end{tabular}

The line diagram of $1^{\text {st }}$ difference for each company is shown in Figure 2.

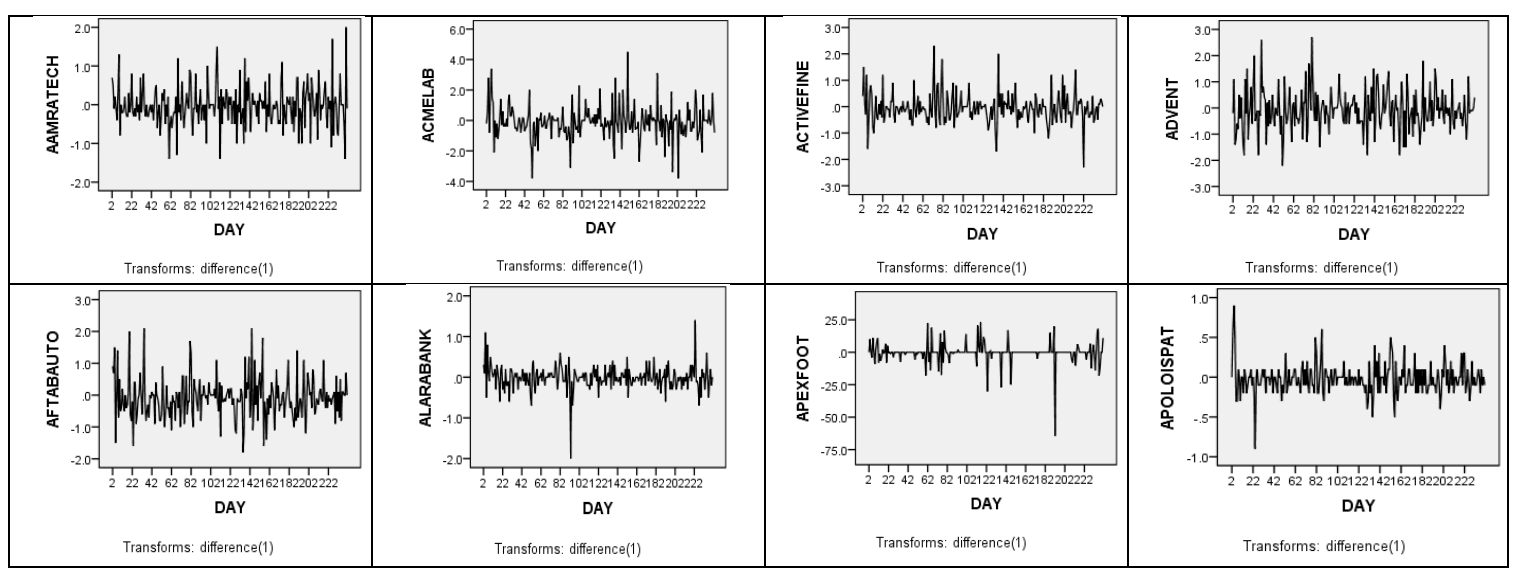




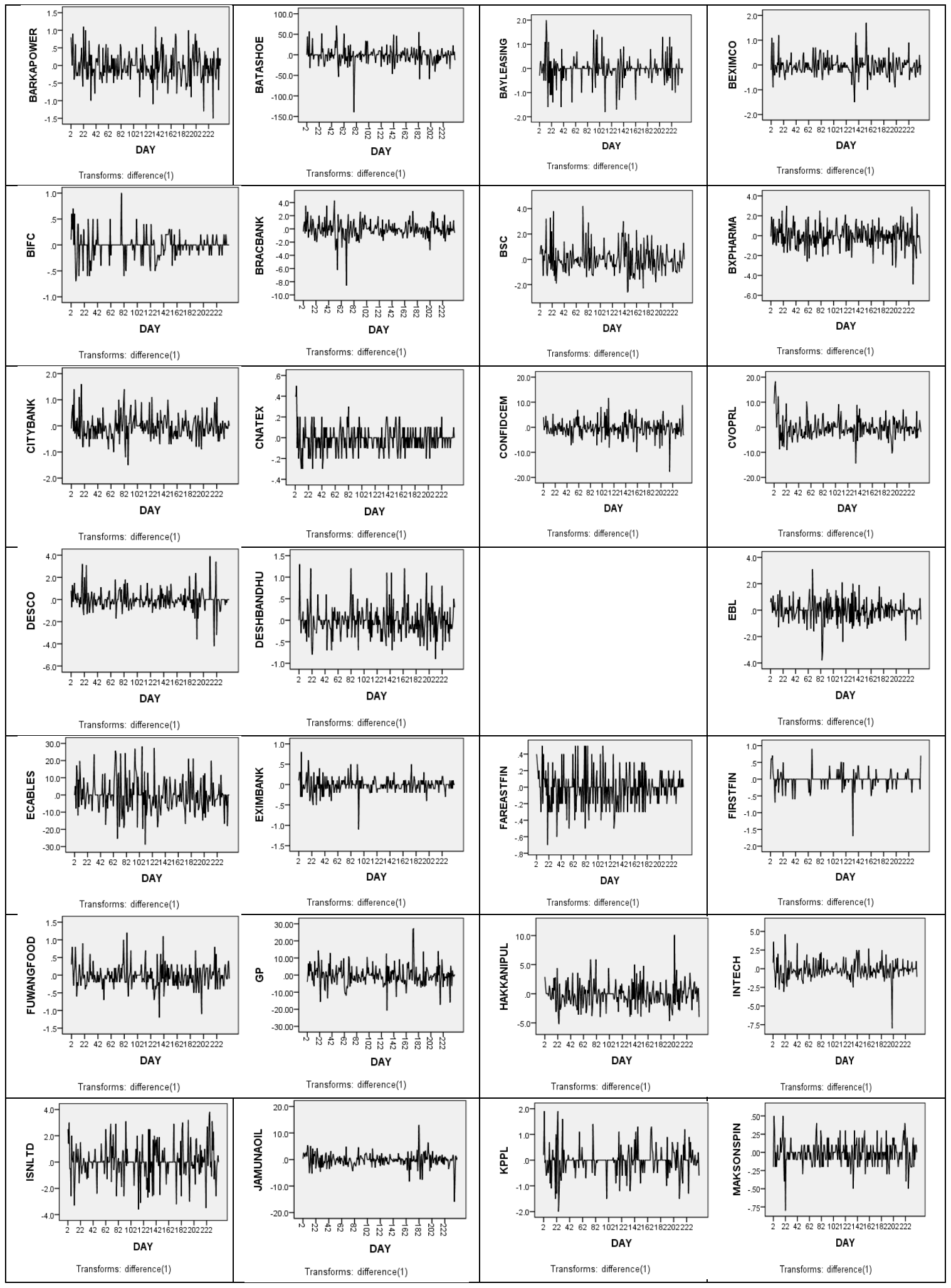




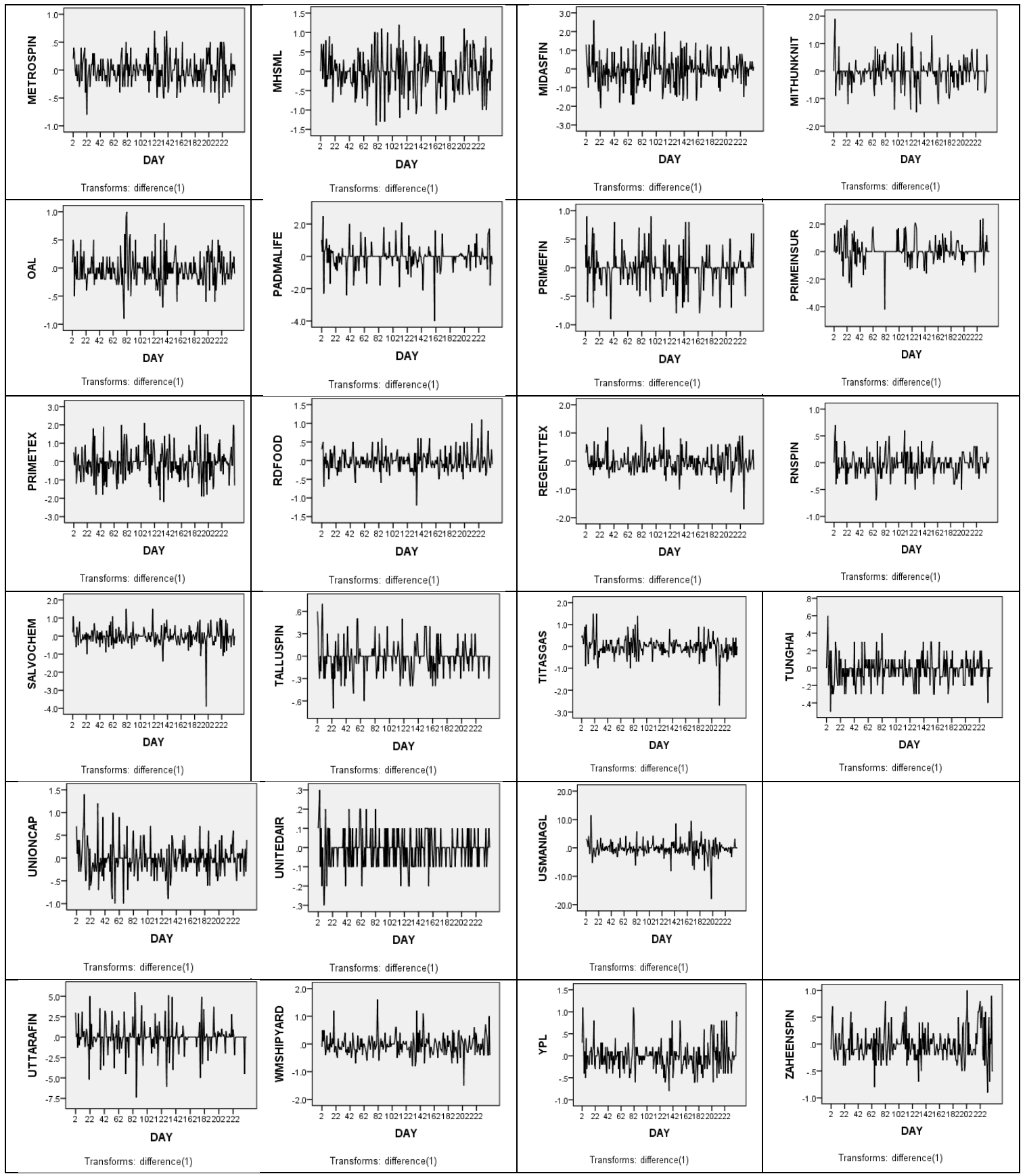

Figure 2: Line diagram of $1^{\text {st }}$ difference

The diagram for autocorrelation function (ACF)oflag 16 foreach share is created to select MA lag value in ARIMA model and result is shown in Figure 3.
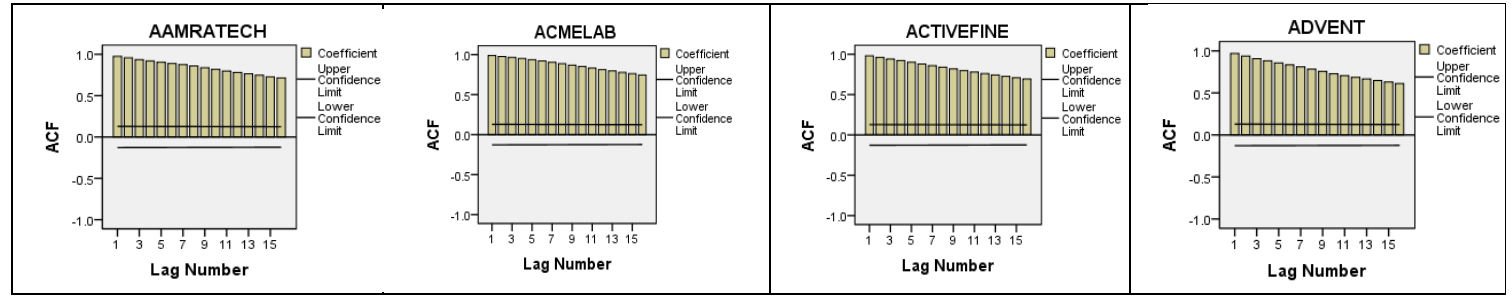


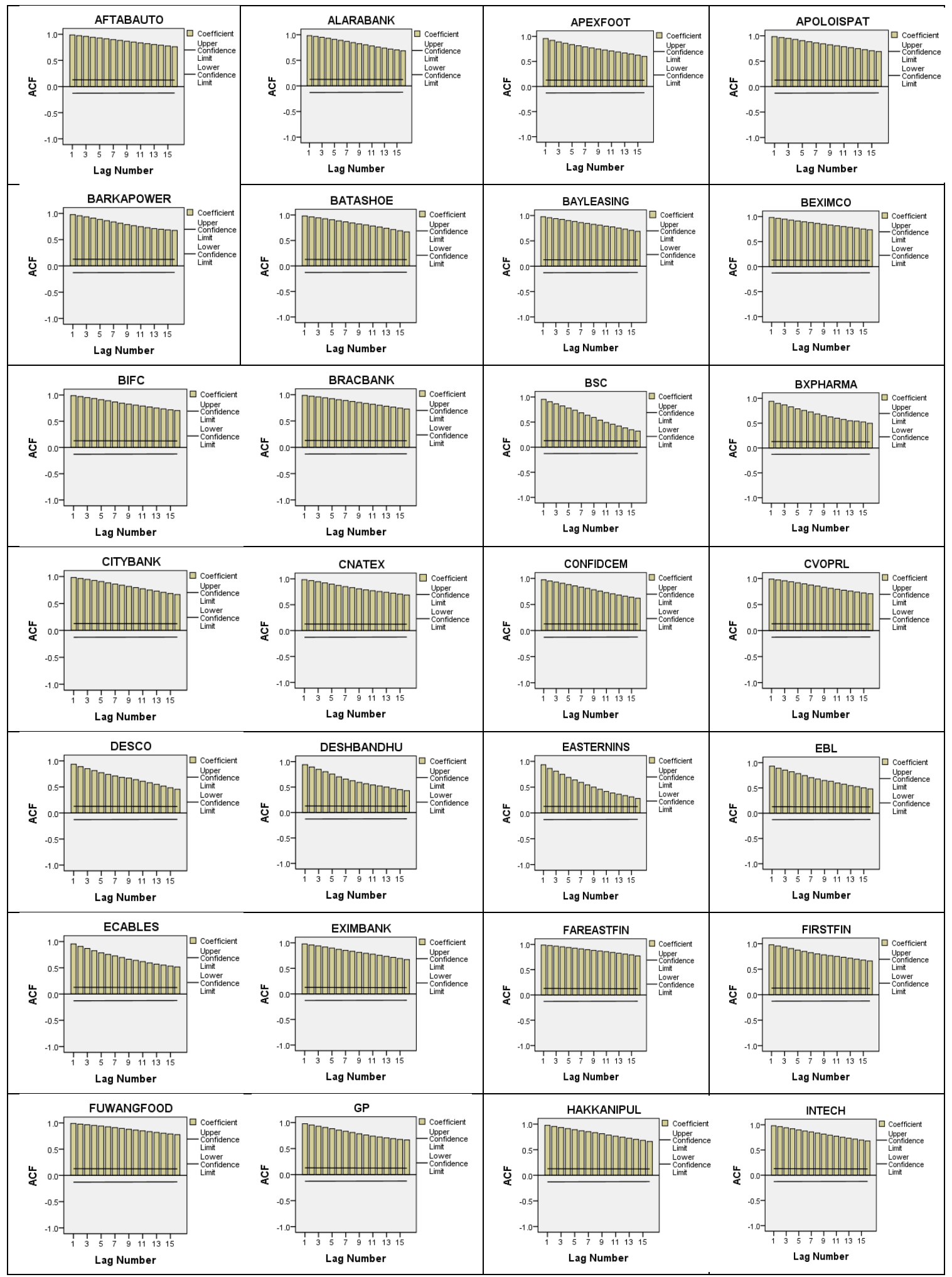




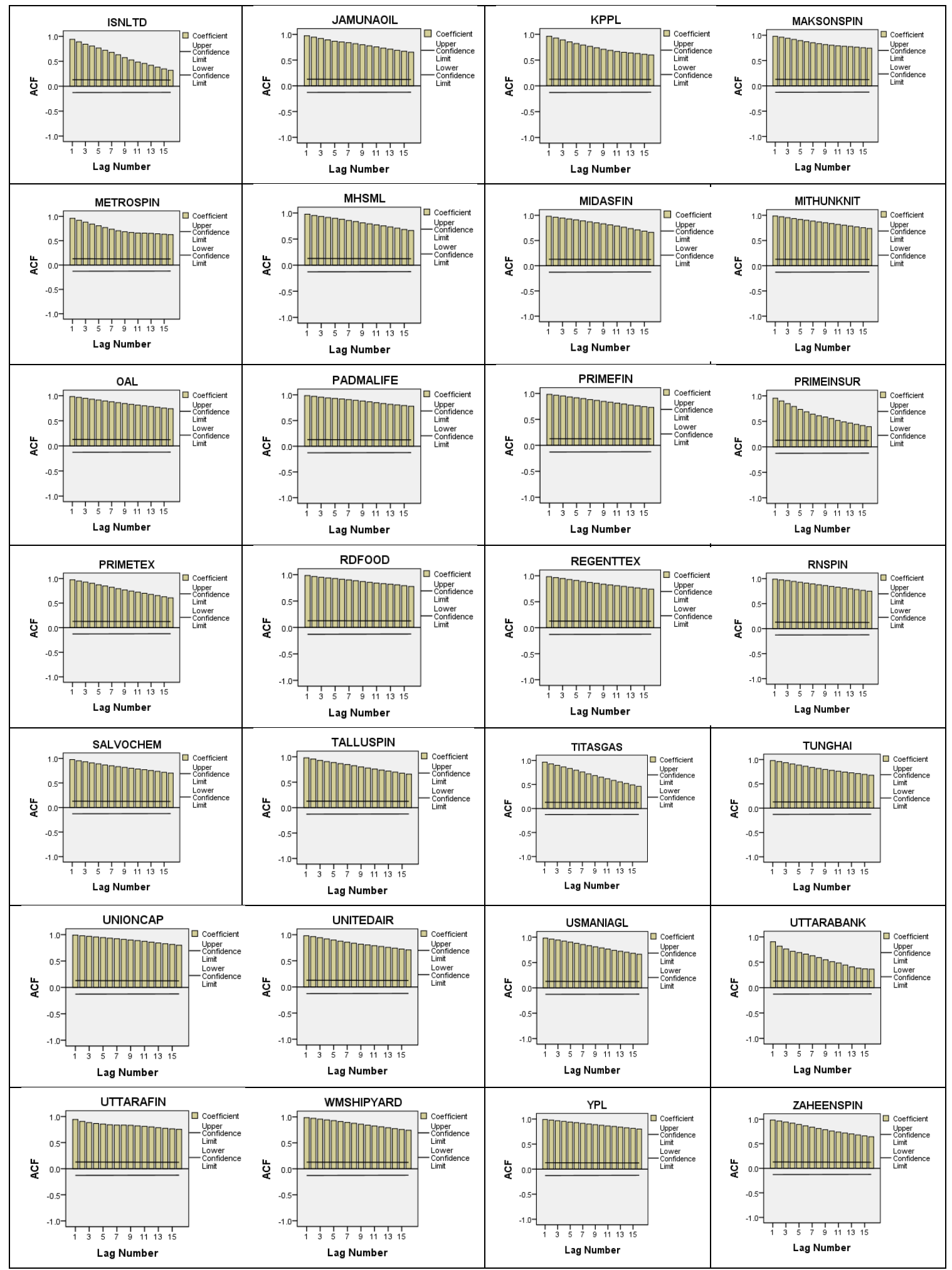

Figure 3: ACF diagram of share price

From the above figure it is seen that, the autocorrelation factors decreasesgradually and all the values are higher than the confidence interval for all cases. So, no moving average slag value is convenient for the model development. It is convenient to consider MA(0) for the model of each company daily share price. Now the diagram for partial autocorrelation function (PACF) of lag 16 foreach share is created to select 
AR lag value in ARIMA model and result is shown in Figure

4.

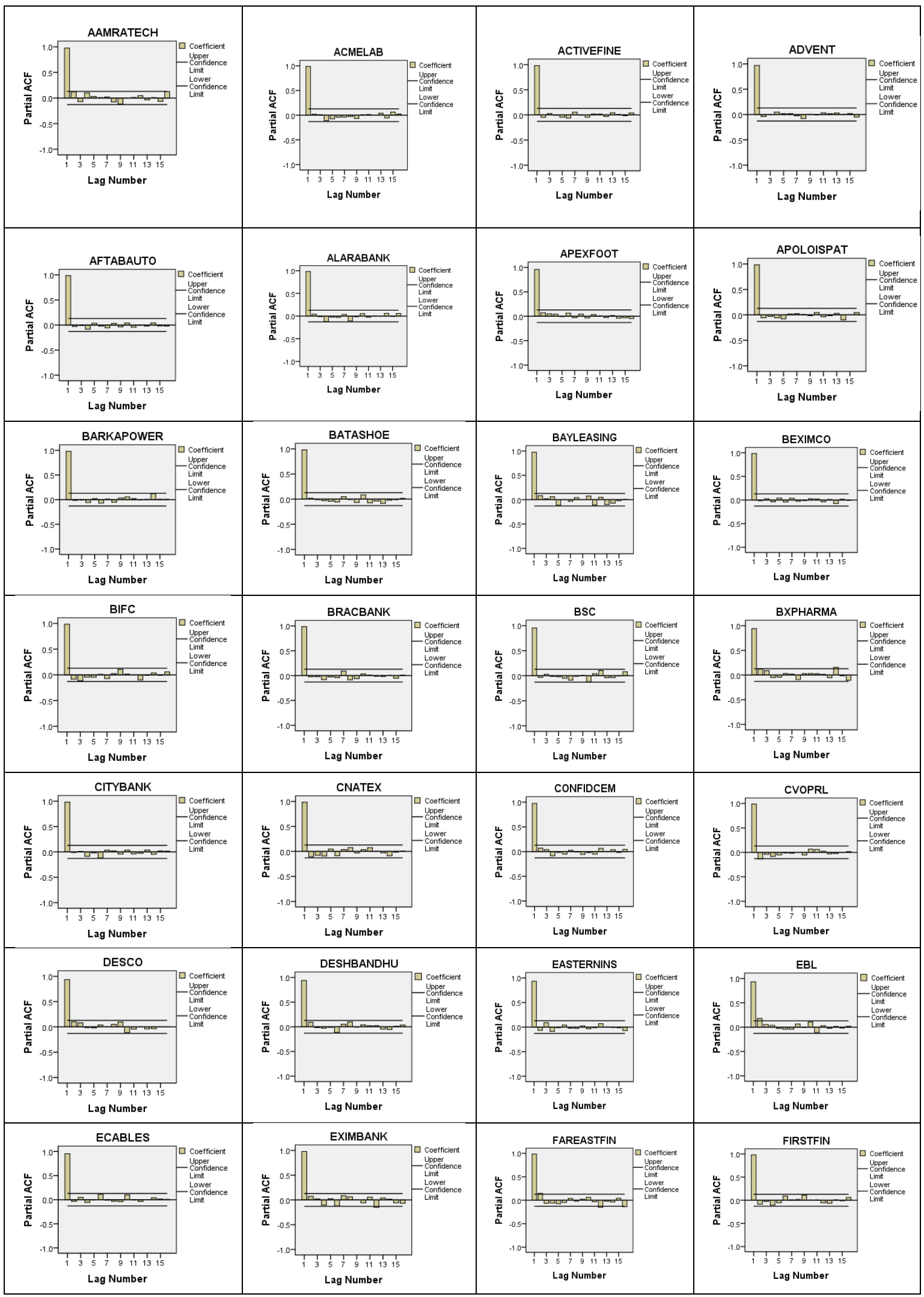




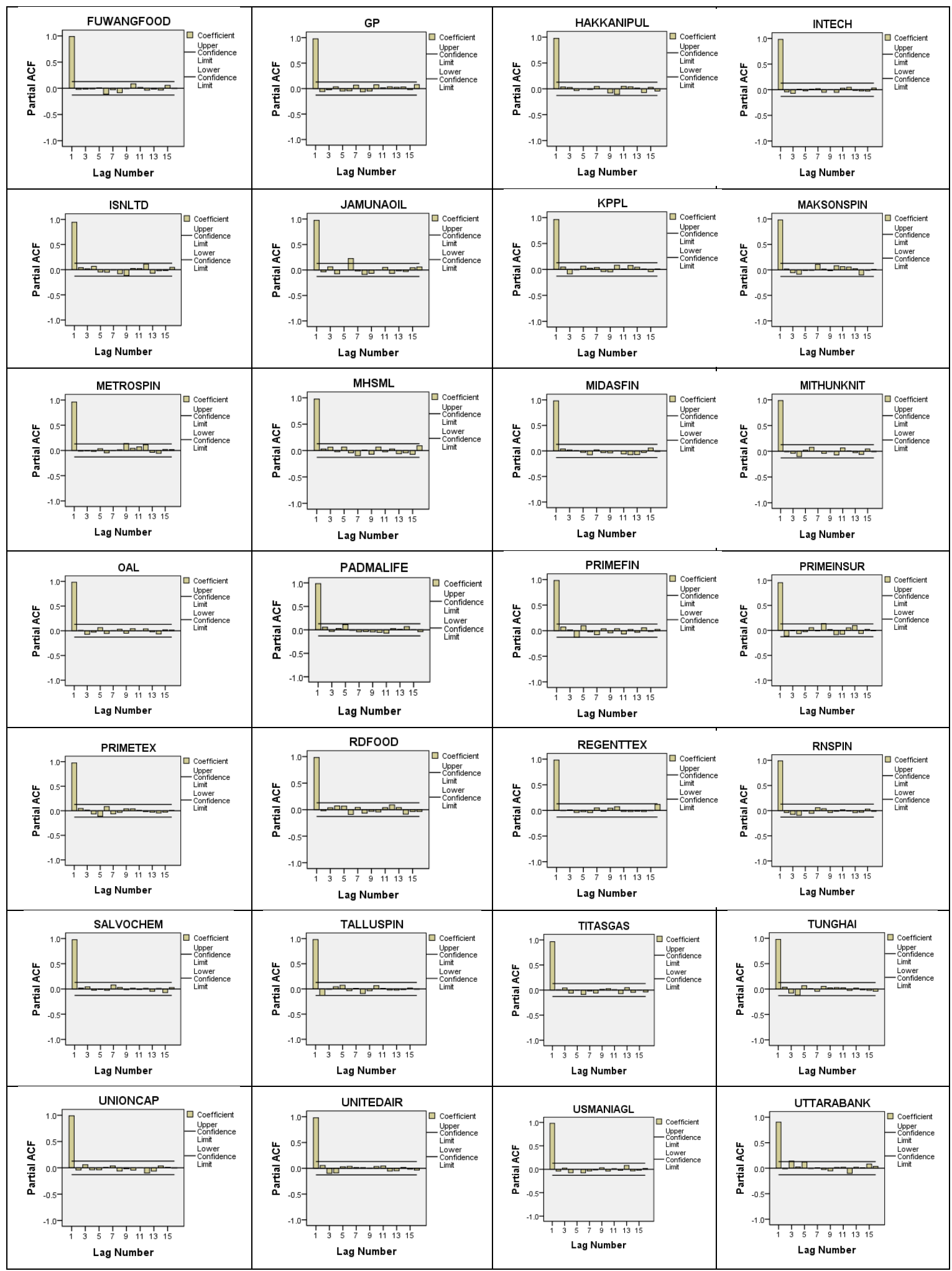



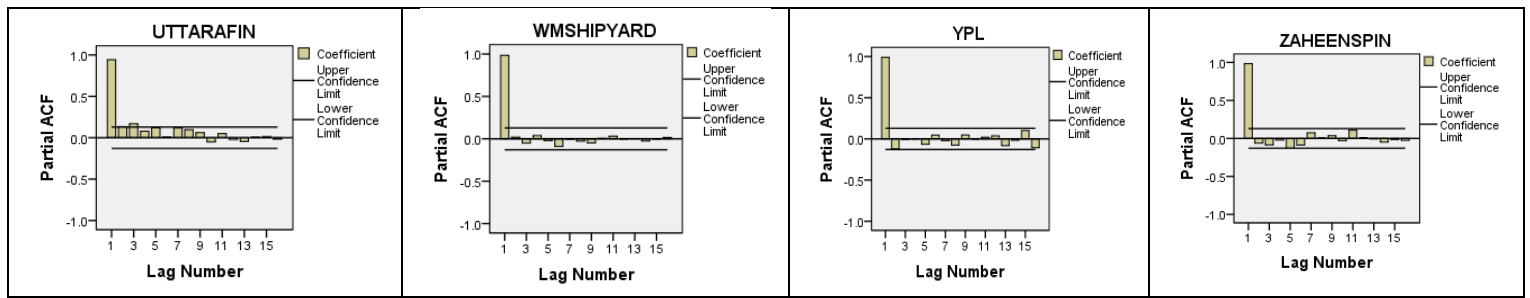

Figure 4: PACF diagram of share price

From the above figures the number of partial autocorrelation values that higher than the confidence interval has been selected for AR(p) model. Then, based on the January 2019 to December 2019 stock prices (241 trading days) data with AR, I and MA values of 52 companies $(86.67 \%)$ are selected for ARIMA $(1,1,0), 4$ companies $(6.67 \%)$ are selected for
ARIMA (2,1,0), 1 company (1.67\%) selected for ARIMA $(3,1,0), 1$ company $(1.67 \%)$ is selected for ARIMA $(4,1,0), 1$ company $(1.67 \%)$ is selected for ARIMA $(1,0,0)$ and 1 company $(1.67 \%)$ is selected for ARIMA $(2,1,0)$ model. The result is shown in Table 1.The coefficient of each selected ARIMA model is calculated and shown in Table II.

TABLE IIARIMA model for share price

\begin{tabular}{|c|c|c|c|c|c|c|}
\hline Variable Name & ARIMA model & $\begin{array}{c}\text { Ljung-Box Test } \\
\text { Q (p-value) }\end{array}$ & RMSE & MAE & MAPE & $\begin{array}{l}\text { R-Square } \\
\text { value }\end{array}$ \\
\hline AAMRATECH & $\begin{aligned} & \mathrm{Y}_{\mathrm{t}}=- 0.028-0.217 \mathrm{Y}_{(\mathrm{t}-1)} \\
& \text { Difference } 1\end{aligned}$ & $16.049(0.520)$ & 0.508 & 0.369 & 1.479 & 0.965 \\
\hline ACMELAB & $\begin{aligned} & Y_{\mathrm{t}}=- 0.103-0.014 \mathrm{Y}_{(\mathrm{t}-1)} \\
& \text { Difference 1 }\end{aligned}$ & $15.267(0.576)$ & 1.075 & 0.718 & 0.973 & 0.989 \\
\hline ACTIVEFINE & $\begin{array}{c}\mathrm{Y}_{\mathrm{t}}=-0.060+0.062 \mathrm{Y}_{(\mathrm{t}-1)} \\
\text { Difference 1 }\end{array}$ & $19.743(0.288)$ & 0.552 & 0.388 & 1.653 & 0.985 \\
\hline ADVENT & $\begin{array}{c}\mathrm{Y}_{\mathrm{t}}=-0.074+0.026 \mathrm{Y}_{(\mathrm{t}-1)} \\
\text { Difference } 1\end{array}$ & $12.070(0.796)$ & 0.776 & 0.577 & 1.870 & 0.971 \\
\hline AFTABAUTO & $\begin{array}{c}\mathrm{Y}_{\mathrm{t}}=-0.090-0.052 \mathrm{Y}_{(\mathrm{t}-1)} \\
\text { Difference } 1\end{array}$ & $12.645(0.760)$ & 0.637 & 0.455 & 1.311 & 0.992 \\
\hline ALARABANK & $\begin{aligned} & \mathrm{Y}_{\mathrm{t}}=- 0.013-0.080 \mathrm{Y}_{(\mathrm{t}-1)} \\
& \text { Difference } 1\end{aligned}$ & $9.595(0.920)$ & 0.294 & 0.192 & 0.992 & 0.973 \\
\hline APEXFOOT & $\begin{array}{c}\mathrm{Y}_{\mathrm{t}}=-0.302-0.158 \mathrm{Y}_{(\mathrm{t}-1)} \\
\text { Difference } 1\end{array}$ & $12.949(0.740)$ & 7.645 & 3.561 & 1.327 & 0.931 \\
\hline APOLOISPAT & $\begin{array}{c}\mathrm{Y}_{\mathrm{t}}=-0.019+0.128 \mathrm{Y}_{(\mathrm{t}-1)} \\
\text { Difference } 1\end{array}$ & $13.417(0.708)$ & 0.191 & 0.139 & 2.294 & 0.985 \\
\hline BARKAPOWER & $\begin{array}{c}\mathrm{Y}_{\mathrm{t}}=-0.027-0.046 \mathrm{Y}_{(\mathrm{t}-1)} \\
\text { Difference } 1\end{array}$ & $12.931(0.741)$ & 0.410 & 0.313 & 1.133 & 0.975 \\
\hline BATASHOE & $\begin{array}{c}\mathrm{Y}_{\mathrm{t}}=-1.558-0.018 \mathrm{Y}_{(\mathrm{t}-1)} \\
\text { Difference } 1\end{array}$ & $18.942(0.332)$ & 2.285 & 12.435 & 1.260 & 0.981 \\
\hline BAYLEASING & $\begin{array}{c}\mathrm{Y}_{\mathrm{t}}=-0.027-0.112 \mathrm{Y}_{(\mathrm{t}-1)} \\
\text { Difference } 1\end{array}$ & $23.566(0.132)$ & 0.507 & 0.293 & 1.727 & 0.965 \\
\hline BEXIMCO & $\begin{array}{c}\mathrm{Y}_{\mathrm{t}}=-0.049+0.029 \mathrm{Y}_{(\mathrm{t}-1)} \\
\text { Difference } 1\end{array}$ & $16.133(0.514)$ & 0.371 & 0.263 & 1.319 & 0.989 \\
\hline BIFC & $\begin{array}{c}\mathrm{Y}_{\mathrm{t}}=-0.014+0.093 \mathrm{Y}_{(\mathrm{t}-1)} \\
\text { Difference } 1\end{array}$ & $8.795(0.946)$ & 0.221 & 0.130 & 3.023 & 0.982 \\
\hline BRACBANK & $\begin{array}{c}\mathrm{Y}_{\mathrm{t}}=-0.076+0.041 \mathrm{Y}_{(\mathrm{t}-1)} \\
\text { Difference 1 }\end{array}$ & $13.228(0.721)$ & 1.331 & 0.894 & 1.351 & 0.980 \\
\hline $\mathrm{BSC}$ & $\begin{array}{c}\mathrm{Y}_{\mathrm{t}}=0.010+0.025 \mathrm{Y}_{(\mathrm{t}-1)} \\
\text { Difference } 1\end{array}$ & $18.968(0.330)$ & 1.034 & 0.742 & 1.512 & 0.932 \\
\hline BXPHARMA & $\begin{array}{c}\mathrm{Y}_{\mathrm{t}}=-0.053-0.176 \mathrm{Y}_{(\mathrm{t}-1)-} \\
0.111 \mathrm{Y}_{(\mathrm{t}-2)} \\
\text { Difference 1 }\end{array}$ & $11.566(0.773)$ & 1.126 & 0.842 & 1.033 & 0.926 \\
\hline CITYBANK & $\begin{aligned} & \mathrm{Y}_{\mathrm{t}}=- 0.040+0.006 \mathrm{Y}_{(\mathrm{t}-1)} \\
& \text { Difference } 1\end{aligned}$ & $15.099(0.588)$ & 0.452 & 0.334 & 1.265 & 0.982 \\
\hline CNATEX & $\begin{array}{c}\mathrm{Y}_{\mathrm{t}}=-0.010+0.027 \mathrm{Y}_{(\mathrm{t}-1)} \\
\text { Difference } 1\end{array}$ & $12.581(0.764)$ & 0.122 & 0.090 & 3.081 & 0.982 \\
\hline CONFIDCEM & $\begin{array}{c}\mathrm{Y}_{\mathrm{t}}=-0.335-0.193 \mathrm{Y}_{(\mathrm{t}-1)} \\
\text { Difference } 1\end{array}$ & $8.797(0.946)$ & 3.252 & 2.281 & 1.585 & 0.977 \\
\hline CVOPRL & $\begin{array}{c}\mathrm{Y}_{\mathrm{t}}=-0.275+0.241 \mathrm{Y}_{(\mathrm{t}-1)} \\
\text { Difference } 1\end{array}$ & $10.768(0.868)$ & 3.943 & 2.892 & 1.990 & 0.988 \\
\hline DESCO & $\begin{array}{c}\mathrm{Y}_{\mathrm{t}}=-0.012-0.150 \mathrm{Y}_{(\mathrm{t}-1)} \\
\text { Difference } 1\end{array}$ & $18.789(0.341)$ & 0.898 & 0.568 & 1.301 & 0.900 \\
\hline DESHBANDHU & $\begin{array}{c}\mathrm{Y}_{\mathrm{t}}=-0.010-0.125 \mathrm{Y}_{(\mathrm{t}-1)} \\
\text { Difference } 1\end{array}$ & $18.948(0.332)$ & 0.362 & 0.255 & 1.992 & 0.901 \\
\hline EASTERNINS & $\begin{array}{c}\mathrm{Y}_{\mathrm{t}}=-42.662-0.968 \mathrm{Y}_{(\mathrm{t}-1)} \\
\text { Difference } 0\end{array}$ & $12.917(0.742)$ & 2.177 & 1.321 & 2.929 & 0.883 \\
\hline
\end{tabular}




\begin{tabular}{|c|c|c|c|c|c|c|}
\hline Variable Name & ARIMA model & $\begin{array}{c}\text { Ljung-Box Test } \\
\mathrm{Q} \text { (p-value) }\end{array}$ & RMSE & MAE & MAPE & $\begin{array}{c}\text { R-Square } \\
\text { value }\end{array}$ \\
\hline EBL & $\begin{array}{c}\mathrm{Y}_{\mathrm{t}}=-0.014-0.232 \mathrm{Y}_{(\mathrm{t}-1)-} \\
0.101 \mathrm{Y}_{(\mathrm{t}-2)} \\
\text { Difference 1 }\end{array}$ & $22.820(0.119)$ & 0.798 & 0.555 & 1.500 & 0.883 \\
\hline ECABLES & $\begin{array}{c}\mathrm{Y}_{\mathrm{t}}=-0.117-0.013 \mathrm{Y}_{(\mathrm{t}-1)} \\
\text { Difference 1 }\end{array}$ & $18.416(0.363)$ & 9.626 & 6.898 & 2.382 & 0.936 \\
\hline EXIMBANK & $\begin{array}{c}\mathrm{Y}_{\mathrm{t}}=-0.009-0.135 \mathrm{Y}_{(\mathrm{t}-1)-} \\
0.090 \mathrm{Y}_{(\mathrm{t}-2)} \\
\text { Difference 1 }\end{array}$ & $19.538(0.242)$ & 0.185 & 0.123 & 1.085 & 0.965 \\
\hline FAREASTFIN & $\begin{array}{c}\mathrm{Y}_{\mathrm{t}}=-0.010+0.257 \mathrm{Y}_{(\mathrm{t}-1)-} \\
0.003 \mathrm{Y}_{(\mathrm{t}-2)}+0.044 \mathrm{Y}_{(\mathrm{t}-3)}+ \\
0.064 \mathrm{Y}_{(\mathrm{t}-4)} \\
\text { Difference } 1\end{array}$ & $23.602(0.051)$ & 0.208 & 0.155 & 3.591 & 0.980 \\
\hline FIRSTFIN & $\begin{array}{c}\mathrm{Y}_{\mathrm{t}}=-0.007+0.036 \mathrm{Y}_{(\mathrm{t}-1)} \\
\text { Difference } 1\end{array}$ & $15.609(0.552)$ & 0.230 & 0.105 & 2.109 & 0.964 \\
\hline FUWANGFOOD & $\begin{array}{c}\mathrm{Y}_{\mathrm{t}}=-0.023+0.011 \mathrm{Y}_{(\mathrm{t}-1)} \\
\text { Difference 1 }\end{array}$ & $21.457(0.206)$ & 0.318 & 0.229 & 1.770 & 0.987 \\
\hline GP & $\begin{array}{c}\mathrm{Y}_{\mathrm{t}}=-0.346+0.075 \mathrm{Y}_{(\mathrm{t}-1)} \\
\text { Difference 1 }\end{array}$ & $12.312(0.781)$ & 5.839 & 4.054 & 1.171 & 0.971 \\
\hline HAKKANIPUL & $\begin{array}{c}\mathrm{Y}_{\mathrm{t}}=-0.103-0.074 \mathrm{Y}_{(\mathrm{t}-1)} \\
\text { Difference 1 }\end{array}$ & $11.668(0.820)$ & 2.049 & 1.483 & 2.575 & 0.963 \\
\hline INTECH & $\begin{array}{c}\mathrm{Y}_{\mathrm{t}}=-0.185+0.097 \mathrm{Y}_{(\mathrm{t}-1)} \\
\text { Difference 1 }\end{array}$ & $22.665(0.160)$ & 1.200 & 0.808 & 2.798 & 0.991 \\
\hline ISNLTD & $\begin{array}{c}\mathrm{Y}_{\mathrm{t}}=0.065-0.065 \mathrm{Y}_{(\mathrm{t}-1)} \\
\text { Difference 1 }\end{array}$ & $15.249(0.578)$ & 1.312 & 0.859 & 2.734 & 0.912 \\
\hline JAMUNAOIL & $\begin{array}{c}\mathrm{Y}_{\mathrm{t}}=-0.160+0.015 \mathrm{Y}_{(\mathrm{t}-1)-} \\
0.137 \mathrm{Y}_{(\mathrm{t}-2)} \\
\text { Difference 1 }\end{array}$ & $11.850(0.754)$ & 2.517 & 1.702 & 0.991 & 0.968 \\
\hline KPPL & $\begin{array}{c}\mathrm{Y}_{\mathrm{t}}=-0.026-0.089 \mathrm{Y}_{(\mathrm{t}-1)} \\
\text { Difference } 1\end{array}$ & $17.684(0.409)$ & 0.531 & 0.315 & 2.051 & 0.943 \\
\hline MAKSONSPIN & $\begin{array}{c}\mathrm{Y}_{\mathrm{t}}=-0.015-0.004 \mathrm{Y}_{(\mathrm{t}-1)} \\
\text { Difference } 1\end{array}$ & $23.132(0.145)$ & 0.167 & 0.123 & 2.105 & 0.979 \\
\hline METROSPIN & $\begin{array}{c}\mathrm{Y}_{\mathrm{t}}=-0.007-0.013 \mathrm{Y}_{(\mathrm{t}-1)} \\
\text { Difference 1 }\end{array}$ & $16.090(0.517)$ & 0.244 & 0.187 & 2.552 & 0.925 \\
\hline MHSML & $\begin{array}{c}\mathrm{Y}_{\mathrm{t}}=-0.015-0.059 \mathrm{Y}_{(\mathrm{t}-1)} \\
\text { Difference 1 }\end{array}$ & $14.296(0.646)$ & 0.494 & 0.360 & 3.285 & 0.956 \\
\hline MIDASFIN & $\begin{array}{c}\mathrm{Y}_{\mathrm{t}}=-0.047-0.145 \mathrm{Y}_{(\mathrm{t}-1)} \\
\text { Difference 1 }\end{array}$ & $10.308(0.890)$ & 0.765 & 0.551 & 3.029 & 0.973 \\
\hline MITHUNKNIT & $\begin{array}{c}\mathrm{Y}_{\mathrm{t}}=-0.046-0.004 \mathrm{Y}_{(\mathrm{t}-1)} \\
\text { Difference } 1\end{array}$ & $14.501(0.631)$ & 0.455 & 0.286 & 2.344 & 0.987 \\
\hline OAL & $\begin{array}{c}\mathrm{Y}_{\mathrm{t}}=-0.029-0.053 \mathrm{Y}_{(\mathrm{t}-1)} \\
\text { Difference 1 }\end{array}$ & $13.735(0.686)$ & 0.268 & 0.201 & 2.163 & 0.986 \\
\hline PADMALIFE & $\begin{array}{c}\mathrm{Y}_{\mathrm{t}}=-0.037-0.109 \mathrm{Y}_{(\mathrm{t}-1)} \\
\text { Difference 1 }\end{array}$ & $19.532(0.299)$ & 0.701 & 0.394 & 1.902 & 0.975 \\
\hline PRIMEFIN & $\begin{array}{c}\mathrm{Y}_{\mathrm{t}}=-0.007-0.133 \mathrm{Y}_{(\mathrm{t}-1)} \\
\text { Difference } 1\end{array}$ & $12.134(0.792)$ & 0.288 & 0.182 & 2.109 & 0.966 \\
\hline PRIMEINSUR & $\begin{array}{c}\mathrm{Y}_{\mathrm{t}}=0.064+0.149 \mathrm{Y}_{(\mathrm{t}-1)} \\
\text { Difference 1 }\end{array}$ & $17.798(0.402)$ & 0.807 & 0.491 & 2.315 & 0.948 \\
\hline PRIMETEX & $\begin{array}{c}\mathrm{Y}_{\mathrm{t}}=-0.059-0.093 \mathrm{Y}_{(\mathrm{t}-1)} \\
\text { Difference } 1\end{array}$ & $19.347(0.309)$ & 0.814 & 0.590 & 2.155 & 0.968 \\
\hline RDFOOD & $\begin{aligned} \mathrm{Y}_{\mathrm{t}}=- & 0.013-0.022 \mathrm{Y}_{(\mathrm{t}-1)} \\
& \text { Difference 1 }\end{aligned}$ & $26.320(0.069)$ & 0.269 & 0.193 & 1.442 & 0.974 \\
\hline REGENTTEX & $\begin{array}{c}\mathrm{Y}_{\mathrm{t}}=-0.028+0.016 \mathrm{Y}_{(\mathrm{t}-1)} \\
\text { Difference 1 }\end{array}$ & $13.054(0.733)$ & 0.391 & 0.294 & 2.205 & 0.980 \\
\hline RNSPIN & $\begin{array}{c}\mathrm{Y}_{\mathrm{t}}=-0.021+0.0145 \mathrm{Y}_{(\mathrm{t}-1)} \\
\text { Difference } 1\end{array}$ & $11.213(0.845)$ & 0.201 & 0.149 & 2.735 & 0.990 \\
\hline SALVOCHEM & $\begin{array}{c}\mathrm{Y}_{\mathrm{t}}=-0.035-0.065 \mathrm{Y}_{(\mathrm{t}-1)} \\
\text { Difference } 1\end{array}$ & $11.557(0.826)$ & 0.494 & 0.318 & 2.382 & 0.970 \\
\hline TALLUSPIN & $\begin{array}{c}\mathrm{Y}_{\mathrm{t}}=-0.014+0.083 \mathrm{Y}_{(\mathrm{t}-1)} \\
\text { Difference } 1\end{array}$ & $11.213(0.845)$ & 0.198 & 0.127 & 2.623 & 0.975 \\
\hline TITASGAS & $\begin{array}{c}\mathrm{Y}_{\mathrm{t}}=-0.024-0.047 \mathrm{Y}_{(\mathrm{t}-1)} \\
\text { Difference 1 }\end{array}$ & $19.936(0.277)$ & 0.434 & 0.301 & 0.804 & 0.964 \\
\hline TUNGHAI & $\begin{array}{c}\mathrm{Y}_{\mathrm{t}}=- \\
\text { Difference 1 }\end{array}$ & $15.249(0.578)$ & 0.149 & 0.106 & 3.087 & 0.981 \\
\hline UNIONCAP & $\begin{array}{c}Y_{t}=-0.026-0.062 Y_{(t-1)} \\
\text { Difference 1 }\end{array}$ & $19.934(0.278)$ & 0.345 & 0.237 & 2.378 & 0.987 \\
\hline UNITEDAIR & $\begin{array}{c}\mathrm{Y}_{\mathrm{t}}=- \\
\text { Difference 1 }\end{array}$ & 7.264(0.980) & 0.089 & 0.064 & 3.014 & 0.978 \\
\hline
\end{tabular}




\begin{tabular}{|c|c|c|c|c|c|c|}
\hline Variable Name & ARIMA model & $\begin{array}{c}\text { Ljung-Box Test } \\
\mathrm{Q} \text { (p-value) }\end{array}$ & RMSE & MAE & MAPE & $\begin{array}{l}\text { R-Square } \\
\text { value }\end{array}$ \\
\hline USMANIAGL & $\begin{array}{c}\mathrm{Y}_{\mathrm{t}}=-0.232+0.041 \mathrm{Y}_{(\mathrm{t}-1)} \\
\text { Difference 1 }\end{array}$ & $19.192(0.318)$ & 2.740 & 1.740 & 2.006 & 0.983 \\
\hline UTTARABANK & $\begin{array}{c}\mathrm{Y}_{\mathrm{t}}=28.411+0.907 \mathrm{Y}_{(\mathrm{t}-1)^{-}} \\
0.001 \mathrm{Y}_{(\mathrm{t}-2)} \\
\text { Difference } 0\end{array}$ & $16.691(0.406)$ & 0.482 & 0.332 & 1.154 & 0.823 \\
\hline UTTARAFIN & $\begin{array}{c}\mathrm{Y}_{\mathrm{t}}=-0.049-0.261 \mathrm{Y}_{(\mathrm{t}-1)}- \\
0.246 \mathrm{Y}_{(\mathrm{t}-2)-}-0.126 \mathrm{Y}_{(\mathrm{t}-3)} \\
\text { Difference 1 }\end{array}$ & $14.613(0.480)$ & 1.619 & 1.043 & 1.696 & 0.907 \\
\hline WMSHIPYARD & $\begin{array}{c}\mathrm{Y}_{\mathrm{t}}=-0.040-0.002 \mathrm{Y}_{(\mathrm{t}-1)} \\
\text { Difference } 1\end{array}$ & $14.940(0.600)$ & 0.357 & 0.260 & 1.726 & 0.988 \\
\hline YPL & $\begin{array}{c}\mathrm{Y}_{\mathrm{t}}=-0.005+0.112 \mathrm{Y}_{(\mathrm{t}-1)} \\
\text { Difference } 1\end{array}$ & $14.609(0.624)$ & 0.321 & 0.230 & 2.105 & 0.982 \\
\hline ZAHEENSPIN & $\begin{array}{c}\mathrm{Y}_{\mathrm{t}}=-0.010+0.081 \mathrm{Y}_{(\mathrm{t}-1)} \\
\text { Difference 1 }\end{array}$ & $20.265(0.261)$ & 0.296 & 0.222 & 2.533 & 0.972 \\
\hline
\end{tabular}

The Ljung-Box Test Q-statistics value varies from 7.264 to 26.320 (p-value 0.980 to 0.051 ). For every ARIMA model Ljung-Box Testp-values are greater than 0.05 . The root mean square error (RMSE) values of the ARIMA model varies from 0.089 to 0.626 . The mean absolute error value of the model also varies from 0.064 to 6.989 . The mean absolute percentage error (MAPE) of each model varies from
$0.804 \%$ to $3.591 \%(<5 \%)$, which shows as excellent fit. On the other hand, the R-Square values of the models are 0.823 to 0.992 . So, $82.3 \%$ to $99.2 \%$ data are fit in the developed ARIMA model. By using the above ARIMA models the forecasted share values with confidence interval of January 2020 (22 trading days) for each company are calculated and result is shown in the following Figure 5.

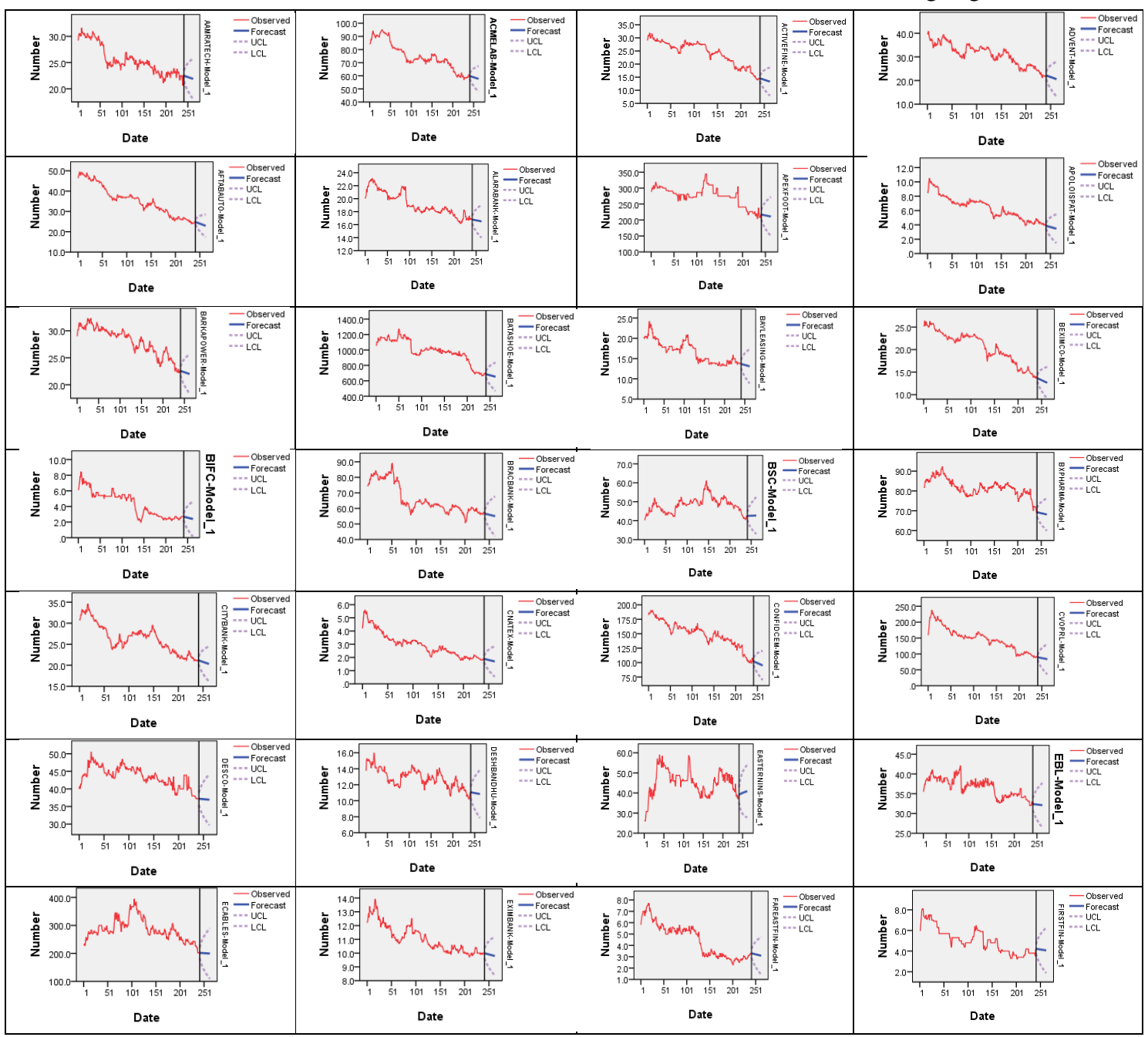




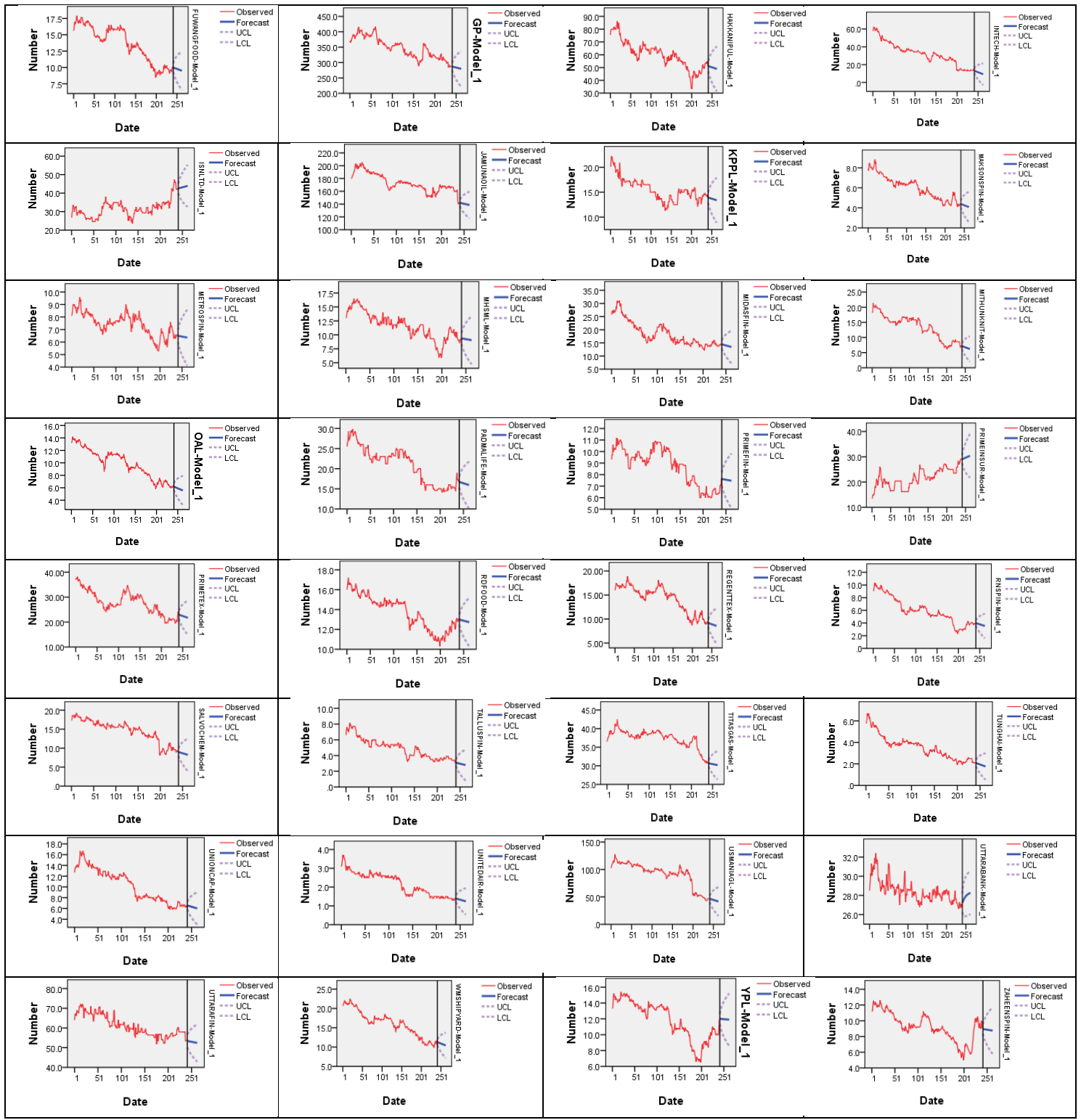

Figure 5. Forecasted value of ARIMA model

After calculating the predicted values, the actual share values of January 2020 (22 trading days) are compared with goodness fit test and result is shown in Table III.

TABLE III ARIMA Model validate of share price

\begin{tabular}{|c|c|c|c|c|}
\hline Variable Name & $\begin{array}{c}\text { Chi-Square of } \\
\text { Goodness fit } \\
\text { test statistic } \\
\text { value (p-value) }\end{array}$ & RMSE & MAPE & MSE \\
\hline AAMRATECH & $6.065(0.999)$ & 2.639 & 9.817 & 6.963 \\
\hline ACMELAB & $2.771(1.000)$ & 2.799 & 3.672 & 7.833 \\
\hline ACTIVEFINE & $0.830(1.000)$ & 0.712 & 4.619 & 0.506 \\
\hline ADVENT & $1.558(1.000)$ & 1.234 & 4.699 & 1.523 \\
\hline AFTABAUTO & $1.211(1.000)$ & 1.158 & 3.760 & 1.340 \\
\hline ALARABANK & $0.391(1.000)$ & 0.534 & 2.759 & 0.285 \\
\hline
\end{tabular}

\begin{tabular}{|c|c|c|c|c|}
\hline Variable Name & $\begin{array}{c}\text { Chi-Square of } \\
\text { Goodness fit } \\
\text { test statistic } \\
\text { value (p-value) }\end{array}$ & RMSE & MAPE & MSE \\
\hline APEXFOOT & $25.751(0.216)$ & 6.492 & 6.394 & 27.974 \\
\hline APOLOISPAT & $0.454(1.000)$ & 0.265 & 6.283 & 0.070 \\
\hline BARKAPOWER & $1.045(1.000)$ & 1.036 & 3.775 & 1.073 \\
\hline BATASHOE & $97.943(0.000)$ & 57.968 & 5.872 & 3360.283 \\
\hline BAYLEASING & $0.000(1.000)$ & 0.380 & 2.307 & 0.144 \\
\hline BEXIMCO & $1.647(1.000)$ & 1.036 & 6.150 & 1.072 \\
\hline
\end{tabular}




\begin{tabular}{|c|c|c|c|c|}
\hline Variable Name & $\begin{array}{l}\text { Chi-Square of } \\
\text { Goodness fit } \\
\text { test statistic } \\
\text { value (p-value) }\end{array}$ & RMSE & MAPE & MSE \\
\hline BIFC & $0.370(1.000)$ & 0.195 & 6.847 & 0.038 \\
\hline BRACBANK & $19.523(0.552)$ & 6.512 & 12.267 & 42.402 \\
\hline BSC & $3.736(1.000)$ & 2.837 & 4.590 & 8.051 \\
\hline BXPHARMA & $13.479(0.891)$ & 6.157 & 8.482 & 37.904 \\
\hline CITYBANK & $8.374(0.993)$ & 2.585 & 12.955 & 6.680 \\
\hline CNATEX & $0.000(1.000)$ & 0.094 & 4.238 & 0.009 \\
\hline CONFIDCEM & $19.285(0.567)$ & 9.242 & 8.056 & 85.411 \\
\hline CVOPRL & $2.760(1.000)$ & 3.270 & 2.999 & 10.693 \\
\hline DESCO & $0.848(1.000)$ & 1.186 & 2.690 & 1.406 \\
\hline DESHBANDHU & $0.672(1.000)$ & 0.559 & 4.848 & 0.313 \\
\hline EASTERNINS & $10.736(0.968)$ & 4.094 & 9.213 & 16.764 \\
\hline EBL & $0.862(1.000)$ & 1.109 & 2.458 & 1.230 \\
\hline ECABLES & $6.860(0.998)$ & 7.754 & 2.642 & 60.126 \\
\hline EXIMBANK & $0.000(1.000)$ & 0.266 & 2.267 & 0.071 \\
\hline FAREASTFIN & $0.000(1.000)$ & 0.167 & 4.075 & 0.028 \\
\hline FIRSTFIN & $2.337(1.000)$ & 0.727 & 3.254 & 0.528 \\
\hline FUWANGFOOD & $2.516(1.000)$ & 1.157 & 6.696 & 1.340 \\
\hline GP & $58.831(0.000)$ & 25.509 & 8.157 & 650.730 \\
\hline HAKKANIPUL & $9.319(0.986)$ & 4.322 & 8.314 & 18.681 \\
\hline INTECH & $8.644(0.992)$ & 2.271 & 5.247 & 5.160 \\
\hline ISNLTD & $9.317(0.986)$ & 4.010 & 9.192 & 16.080 \\
\hline JAMUNAOIL & $1.596(1.000)$ & 3.154 & 1.865 & 9.947 \\
\hline KPPL & $0.815(1.000)$ & 0.738 & 3.864 & 0.544 \\
\hline MAKSONSPIN & $0.644(1.000)$ & 0.373 & 5.890 & 0.139 \\
\hline METROSPIN & $1.353(1.000)$ & 0.589 & 8.726 & 0.347 \\
\hline MHSML & $3.403(1.000)$ & 1.299 & 10.364 & 1.687 \\
\hline MIDASFIN & $1.452(1.000)$ & 0.907 & 5.862 & 0.823 \\
\hline MITHUNKNIT & $8.536(0.992)$ & 1.836 & 9.599 & 3.373 \\
\hline OAL & $0.559(1.000)$ & 0.399 & 5.964 & 0.159 \\
\hline PADMALIFE & $1.053(1.000)$ & 0.890 & 3.854 & 0.792 \\
\hline PRIMEFIN & $1.371(1.000)$ & 0.646 & 8.251 & 0.417 \\
\hline PRIMEINSUR & $1.424(1.000)$ & 1.371 & 3.887 & 1.879 \\
\hline PRIMETEX & $1.350(1.000)$ & 1.127 & 4.337 & 1.269 \\
\hline
\end{tabular}

\begin{tabular}{|c|c|c|c|c|}
\hline Variable Name & $\begin{array}{c}\text { Chi-Square of } \\
\text { Goodness fit } \\
\text { test statistic } \\
\text { value (p-value) }\end{array}$ & RMSE & MAPE & MSE \\
\hline RDFOOD & $1.230(1.000)$ & 0.879 & 5.219 & 0.772 \\
\hline REGENTTEX & $0.420(1.000)$ & 0.396 & 3.485 & 0.157 \\
\hline RNSPIN & $0.454(1.000)$ & 0.257 & 4.978 & 0.066 \\
\hline SALVOCHEM & $3.067(1.000)$ & 1.179 & 10.234 & 1.390 \\
\hline TALLUSPIN & $0.436(1.000)$ & 0.252 & 7.006 & 0.063 \\
\hline TITASGAS & $0.492(1.000)$ & 0.828 & 2.291 & 0.686 \\
\hline TUNGHAI & $0.759(1.000)$ & 0.278 & 10.442 & 0.077 \\
\hline UNIONCAP & $2.266(1.000)$ & 0.739 & 12.092 & 0.546 \\
\hline UNITEDAIR & $1.046(1.000)$ & 0.292 & 12.365 & 0.085 \\
\hline USMANIAGL & $2.214(1.000)$ & 2.102 & 4.217 & 4.418 \\
\hline UTTARABANK & $2.588(1.000)$ & 1.732 & 5.734 & 2.999 \\
\hline UTTARAFIN & $2.159(1.000)$ & 2.244 & 3.937 & 5.034 \\
\hline WMSHIPYARD & $2.978(1.000)$ & 1.287 & 10.032 & 1.657 \\
\hline YPL & $2.539(1.000)$ & 1.106 & 9.406 & 1.224 \\
\hline ZAHEENSPIN & $3.127(1.000)$ & 1.038 & 11.804 & 1.077 \\
\hline
\end{tabular}

In the Chi-Square values of goodness fit test for BATASHOE and GP are 97.943 and 58.831 respectively with p-value 0.000.For these two companies(3.33\%), null hypothesis 3 can be rejected, which implies that there is a significant difference between the actual share price and forecasted share price. For the rest of the 58 companies the goodness of fit tests the ChiSquare values are 0.000 to 25.751 with p-value 1.000 to 0.216 . As a result, for these 58 companies, the null hypothesis 3 can be accepted. This implies that, there is no significant difference between the actual share price and forecasted share price for these 58 companies $(96.67 \%)$. The root mean square error (RMSE) values, the mean absolute percentage error values and mean absolute error values of these 58 companies are varies from 0.094 to $9,242,1.865$ to 12.955 and 0.009 to 85.411 respectively. Finally, it can be seen that, out of these 58 companies 50 companies $(83.33 \%)$ follow $\operatorname{ARIMA}(1,1,0)$ model, 4 companies $(6.67 \%)$ follow $\operatorname{ARIMA}(2,1,0)$ model, 1 company (1.67\%) follow ARIMA(3,1,0)model, 1 company (1.67\%) follow ARIMA(4,1,0)model, 1 company (1.67\%) follows $\operatorname{ARIMA}(1,0,0)$ model and 1 company (1.67\%) follows ARIMA $(2,0,0)$ model.

Moreover, the significance level values of Chi-Square of goodness fit test are greater than 0.05 implies that, there is no significant difference between the predicted stock values and actual stock values. Therefore, it may be concluded saying that ARIMA $(1,1,0)$ model is the most suitable model that can 
be applied to predict the forecast of daily share price in Chittagong Stock Exchange (CSE).

\section{CONCLUSION}

It is observed from the Durbin-Watson test result that there is autocorrelation in the daily current share prices with their previous share prices. The Augmented Dickey-Fuller Test result shows, the daily share prices of $58(96.67 \%)$ selected company data have unit roots. Now, these 58 company's share values are differencing from the previous values $\left(1^{\text {st }}\right.$ differencing) of respective company. The autocorrelation function (ACF) of each share shows that it is convenient to consider MA(0) for the respective ARIMA model. The partial autocorrelation function (PACF) of each share shows that autocorrelation $\operatorname{AR}(1), \operatorname{AR}(2), \operatorname{AR}(3)$ and $\operatorname{AR}(4)$ has been considered for the ARIMA model. Now, from the data of January 2019 to December 2019 daily stock prices (241 trading days) ARIMA $(1,1,0), \operatorname{ARIMA}(2,1,0), \operatorname{ARIMA}(3,1,0)$, $\operatorname{ARIMA}(4,1,0), \operatorname{ARIMA}(1,0,0)$ and $\operatorname{ARIMA}(2,0,0)$ model are selected based on Ljung-Box Test $\mathrm{Q}$, root mean square error, mean absolute error, mean absolute percent error and Rsquare values. By using the above ARIMA models January 2020 (22 trading day) forecasted share values with confidence interval are calculated. These predicted share values are compared with the actual share price of January 2020 (22 trading day) of the respective company and validate with ChiSquare of Goodness fit test, root mean square error, mean absolute percent error, mean square error values. The result shows that 50 companies $(83.33 \%)$ follow $\operatorname{ARIMA}(1,1,0)$ model, 4 companies $(6.67 \%)$ follow $\operatorname{ARIMA}(2,1,0)$ model, 1 company (1.67\%) follow $\operatorname{ARIMA}(3,1,0)$ model, 1 company (1.67\%) follows ARIMA(4,1,0)model, 1 company (1.67\%) follows $\operatorname{ARIMA}(1,0,0)$ model and 1 company (1.67\%) follows ARIMA $(2,0,0)$ model. Finally, it may be concluded that the above ARIMA model may be applied to predict the forecast the daily share prices of Chittagong Stock Exchange (CSE).

\section{REFERENCES:}

[1] Ahmed, F. (2002). Market Efficiency in Emerging Stock Markets: The Case of Dhaka Stock Exchange. Journal of Business Studies, 23 (1), pp. 157-172.

[2] Alam, M. M., Uddin, M. G. S. (2007). The Impacts of Interest Rate on Stock Market: Empirical Evidence from Dhaka Stock Exchange. South Asian Journal of Management and Sciences, Vol. 1(2), pp. 123-132.

[3] Al-Shaib, M. (2006). The predictability of the Amman Stock Exchange Using Univariate Autoregressive Integrated Moving Average (ARIMA) Model. Journal of Economic \& Administrative Sciences, 22, (2), 17-35.

[4] Al-Zeaud, H. A., (2011). Modelling and Forecasting Volatility Using ARIMA Model. European Journal of Economics, Finance and Administrative Sciences, 35, 109-125.

[5] Ariyo, A. A., Adewumi, A. O., and Ayo, C. K. (2014). Stock price prediction using the arima model. in Computer Modelling and Simulation (UKSim) in 2014 UKSim-AMSS $16^{\text {th }}$ International Conference, pages 106-112. IEEE.

[6] Bepari, M.K. and Mollik, A. (2008). Bangladesh Stock Market Growing? a key indicators based assessment. Journal of Business Administration Online (JBAO), Issue 8, 2008, Arkansas: School of Business, Arkansas Tech University.
[7] Cao, L. J., Tay, F. E.H. (2003). Support vector machine with adaptive parameters in financial time series forecasting. IEEE Transactions on Neural Networks, 14: 1506-1518.

[8] Chang, P. C., Liu, C.H. (2008). A TSK type fuzzy rule based system for stock price prediction. Expert Systems with Applications. 34: 135-144.

[9] Claessens, S., Dasgupta S. and Glen, J. (1995). Return behaviour in emerging Stock Market. The world Bank economic Review, vol.9, no.1, Pp. 131-151.

[10] CSE (2021). Chittagong Stock Exchange. Retrieved from https://www.cse.com.bd/market/close price

[11] Ding, Z., Granger,C. W. J., and Engle,R. F. (1993). A long memory property of stock market returns and a new model. Journal of Empirical Finance. 1, 83-106.

[12] Fama, E. F., French, K. R. (1988). Dividend yields and expected stock returns. Journal of financial economics, 22(1), 3-25.

[13] Gavrishchaka, V. V., Banerjee, S. (2006). Support vector machine as an efficient framework for stock market volatility forecasting. Computational Management Science. 3: 147-160.

[14] Granger, M., Morgenstern, O. (1963). Spectral Analysis of New York Stock Market Prices. Kyklos, 16: 1-27

[15] Haider, A. S., Kabir, M. R. (2009). Forecasting Dhaka Stock Exchange (DSE) Return: An Autoregressive Integrated Moving Average (ARIMA) Approach. North South Business Review, Volume 3.

[16] Harvey, C. R., (1994). Conditional Asset allocation in Emerging Markets. Working Paper. No. 4623, Cambridge, MA.

[17] Islam, A., Khaled, M. (2005). Tests of Weak-Form Efficiency of the Dhaka Stock Exchange. Journal Of Business Finance \& Accounting, vol.32 (7-8), pp.1613-1624, September/October.

[18] Jarrett, J.E., and E. Kyper, (2005). Daily variation, capital market efficiency and predicting stock market returns. Management Research News. 28(8),34-47.

[19] Jia, H. (2016). Investigation into the effectiveness of long short term memory networks for stock price prediction. arXiv preprint arXiv:1603.07893.

[20] Kamruzzaman, M., Khudri, M. M. and Rahman, M. M. (2017). Modeling and Predicting Stock Market Returns: A Case Study on Dhaka Stock Exchange of Bangladesh. Dhaka Univ. J. Sci. 65(2): 97-101, 2017 (July)

[21] Kendall, M. (1953). The Analysis of Economic Time Series. Part I. Prices. Journal of the Royal Statistical Society, 96: 11-25.

[22] Keane, S. M. (1983). The Efficient Market Hypothesis on Trial. Financial Analysts Journal. Vol. 42, No. 2 (Mar. - Apr., 1986), pp. 58-63

[23] Kader, A. A., Rahman, A. F. M A. (2005). Testing the WeakForm Efficiency of an Emerging Market: Evidence from the Dhaka Stock Exchange of Bangladesh. AIUB Journal, vol.4 (2), August.

[24] Khababa, N. (1998). Behavior of stock prices in the Saudi Arabian Financial Market: Empirical research findings. Journal of Financial Management \& Analysis. vol.11(1), pp.48-55, Jan-June.

[25] Kryzanowski, L., Galler, M., and Wright, D. W. (1993). Using artificial neural networksto pick stocks. Financial Analysts Journal, 49(4):21-27.

[26] Kumar, S. S. (2006). Forecasting Volatility - Evidence from Indian Stock and Forex Markets. IIM Working Paper series, 6

[27] Liu, C.F., Yeh, C.Y. and Lee, S.J. (2012). Application of type-2 neuro-fuzzy modeling in stock price prediction. Applied Soft Computing. 12: 1348-1358.

[28] Mostafa, M. (2010). Forecasting stock exchange movements using neural networks: empirical evidence from Kuwait. Expert Systems with Application, 37(9), 6302-6309. DOI: 10.1016/j.eswa.2010.02.091.

[29] Oh, S. K., Pedrycz, W., Park, H. S. (2006). Genetically optimized fuzzy polynomial neural networks. IEEE Transactions on Fuzzy Systems. 14: 125-144.

[30] Poterba, J. M., Summers, L. H. (1988). Mean Reversion in Stock Returns: Evidence and Implications. Journal of Financial Economics, Vol. 22, pp. 27-59. 
[31] Rahman, S. \& Hossain, F. (2006). Weak-Form Efficiency: Testimony of Dhaka Stock Exchange. Journal of Business Research, 8, pp.1-12.

[32] Roux, F. J. P., Gilbertson, D. P. (1978). The behavior of share prices on the Johannesburg Stock Exchange. Journal of Business Finance and Accounting. vol.5 (2), pp.223-232

[33] Simons, D., Laryea, S.A. (2004). Testing the Efficiency of selected African Stock Markets. A Working Paper. http://paper.ssrn.com/so13/paper.cfm?abstract_id=874808.

[34] Schöneburg, E. (1990). Stock price prediction using neural networks: A project report. Neurocomputing, 2(1):17-27.

[35] Sen, J. \&Datta Chaudhuri, T. (2018a). Understanding the sectors of Indian economy for portfolio choice. International Journal of Business Forecasting and Marketing Intelligence, 4(2), 178-222. DOI: 10.1504/IJBFMI.2018.090914

[36] Sen, J. \&Datta Chaudhuri, T. (2018b). Stock price prediction using machine learning and deep learning frameworks. Proceedings of the 6th International Conference on Business Analytics and Intelligence (ICBAI'18), Bangalore, India, December 20-22, 2018.

[37] Sen, J. \&Datta Chaudhuri, T. (2017a). A time series analysisbased forecasting framework for the Indian healthcare sector. Journal of Insurance and Financial Management, 3(1), 66-94.

[38] Sen, J. \&Datta Chaudhuri, T. (2017b). A predictive analysis of the Indian FMCG sector using time series decomposition based approach. Journal of Economics Library, 4(2), 206-226. DOI: http://dx.doi.org/10.1453/jel.v4i2.1282
[39] Sen, J. \& Datta Chaudhuri, T. (2017c). A time series analysisbased forecasting approach for the Indian realty sector. International Journal of Applied Economic Studies, 5(4), 8 - 27.

[40] Sen, J. \& Datta Chaudhuri, T. (2017d). A robust predictive model for stock price forecasting. Proceedings of the $5^{\text {th }}$ International Conference on Business Analytics and Intelligence, Bangalore, India, December 11-13, 2017.

[41] Sen, J. \& Datta Chaudhuri, T. (2016). An alternative framework for time series decomposition and forecasting and its relevance for portfolio choice - a comparative study of the Indian consumer durable and small-cap sector. Journal of Economic Library, 3(2), 303-326.

[42] Sohail, C., Kamal, S., \& Ali, I., (2012). Modelling and volatility analysis of share prices using ARCH and GARCH models. World Applied Sciences Journal. 19(1), 77-82.

[43] Solnik, B., (1973). Note on the Validity of the Random Walk for European Stock Prices. Journal of Finance, 28: 1151-1159.

[44] Wei, L.-Y. (2013). A hybrid model based on ANFIS and adaptive expectation genetic algorithm to forecast TAIEX. Economic Modelling, 33:893-899.

[45] Yeh, C. Y., Huang, C. W. \& Lee, S. J. (2011). A multiple-kernel support vector regression approach for stock market price forecasting. Expert Systems with Applications, 38: 2177-2186.

[46] Yoon, Y., Guimaraes, T., \& Swales, G. (1994). Integrating artificial neural networks with rule-based expert systems. Decision Support Systems, 11(5):497-507. 